\title{
A population genetic window into the past and future of the walleye Sander vitreus: relation to historic walleye and the extinct "blue pike" S. v. "glaucus"
}

Amanda E Haponski and Carol A Stepien ${ }^{*}$

\begin{abstract}
Background: Conserving genetic diversity and local adaptations are management priorities for wild populations of exploited species, which increasingly are subject to climate change, habitat loss, and pollution. These constitute growing concerns for the walleye Sander vitreus, an ecologically and economically valuable North American temperate fish with large Laurentian Great Lakes' fisheries. This study compares genetic diversity and divergence patterns across its widespread native range using mitochondrial $(\mathrm{mt})$ DNA control region sequences and nine nuclear DNA microsatellite ( $\mu$ sat) loci, examining historic and contemporary influences. We analyze the genetic and morphological characters of a putative endemic variant- "blue pike" S. v. "glaucus" -described from Lakes Erie and Ontario, which became extinct. Walleye with turquoise-colored mucus also are evaluated, since some have questioned whether these are related to the "blue pike".

Results: Walleye populations are distinguished by considerable genetic divergence (mean $F_{S T} m t D N A=0.32 \pm 0.01$, $\mu$ sat $=0.13 \pm 0.00$ ) and substantial diversity across their range (mean heterozygosity $m t D N A=0.53 \pm 0.02, \mu s a t=0.68 \pm$ 0.03). Southern populations markedly differ, possessing unique haplotypes and alleles, especially the Ohio/New River population that houses the oldest haplotype and has the most pronounced divergence. Northern formerly glaciated populations have greatest diversity in Lake Erie (mean heterozygosity $m t D N A=0.79 \pm 0.00$, $\mu$ sat $=0.72 \pm 0.01$ ). Genetic diversity was much less in the historic Lake Erie samples from 1923-1949 (mean heterozygosity mtDNA $=0.05 \pm$ 0.01 , $\mu$ sat $=0.47 \pm 0.06$ ) than today. The historic "blue pike" had no unique haplotypes/alleles and there is no evidence that it comprised a separate taxon from walleye. Turquoise mucus walleye also show no genetic differentiation from other sympatric walleye and no correspondence to the "blue pike".

Conclusions: Contemporary walleye populations possess high levels of genetic diversity and divergence, despite habitat degradation and exploitation. Genetic and previously published tagging data indicate that natal homing and spawning site philopatry led to population structure. Population patterns were shaped by climate change and drainage connections, with northern ones tracing to post-glacial recolonization. Southerly populations possess unique alleles and may provide an important genetic reservoir. Allelic frequencies of Lake Erie walleye from 7090 years ago significantly differed from those today, suggesting population recovery after extensive habitat loss, pollution, and exploitation. The historic "blue pike" is indistinguishable from walleye, indicating that taxonomic designation is not warranted.
\end{abstract}

Keywords: Biogeography, Blue pike, Control region, Microsatellites, Sander, Walleye

\footnotetext{
* Correspondence: carol.stepien@utoledo.edu

The Great Lakes Genetics/Genomics Laboratory, Lake Erie Center and Department of Environmental Sciences, The University of Toledo, 6200 Bayshore Road, Toledo, OH 43616, USA
} 


\section{Background}

Species today face many challenges that influence their genetic, phenotypic, and ecological diversity and divergence patterns across space and time. The genetic variation of their component populations comprises the raw material underlying overall and local adaptedness, providing resilience to anthropogenic stressors - such as climate change, habitat alteration and loss, invasive species, and exploitation [1,2]. Notably, declines in genetic diversity may lead to decreased fitness, undermining ability to respond to present and future conditions $[3,4]$. Evaluating comparative and hierarchical levels of genetic composition and differentiation of widespread taxa, as well as their unique or reservoir populations, thus is important for prioritizing conservation management strategies $[5,6]$.

Population genetic patterns of today's temperate taxa resulted from historic and contemporary processes $[7,8]$, regulated by their physiological requirements, habitat connectivity, and dispersal capability $[9,10]$. Broadly distributed taxa display heterogeneous patterns over a breadth of environmental and ecological conditions $[11,12]$. Isolated populations experience drift and possibly evolve unique alleles [13,14], whereas large interconnected ones frequently have high gene flow and less genetic distinctiveness $[15,16]$. Low migration may lead to higher divergences, whereas mobility fosters gene flow and homogeneity $[17,18]$.

During the Pleistocene Epoch, 2.6-0.01 million years ago, the North American Laurentide Ice Sheet advanced south to the Ohio River system (Figure 1), altering population distributions and genetic compositions [19,20]. Taxa became sequestered in glacial refugia and then moved northwards to colonize old and new habitats as the ice retreated [21,22]. Recent climate warming is accelerating these historic dispersal patterns northward [23,24].

Anthropogenic factors may further modify genetic patterns and force populations into sub-optimal habitats leading to reduced population size, genetic diversity, and/or local adaptation and resulting in possible extirpation [27]. For example, increasing temperatures may reduce population sizes and ranges of temperate cold-water fishes, e.g., lake trout Salvelinus namaycush (Walbaum 1792) and cutthroat trout Oncorhynchus clarkii (Richardson 1836) $[23,28]$. Rising temperatures and decreasing population sizes have been linked to declining genetic diversity through drift and inbreeding in Atlantic salmon Salmo salar Linnaeus 1758 and brown trout S. trutta Linnaeus 1758 [29]. In contrast, warm-water species, such as the walleye Sander vitreus (Mitchill 1818) may broaden their ranges, shifting their distribution centers north [23]. Some outlying populations may fail to adapt, leading to declining genetic variability and extirpation [28], which may particularly affect southerly fringe areas such as the relict North
River, Alabama population of walleye [30] (site Y; Figure 1). Exploitation and other anthropogenic stressors may accelerate diversity loss further [2,31]. The present study analyzes the genetic patterns of walleye, in light of its past and potential future, to evaluate such patterns of variability, isolation, and continuity over space and time.

The walleye is an ecologically and economically valuable fish $[32,33]$ that supports large Great Lakes' fisheries, peaking in Lake Erie [34]. It inhabits slow turbid lakes to fast flowing clear streams throughout much of North America (Figure 1). Historically, it ranged from the Mackenzie River in the Northwest Territories of Canada, south to the U.S. Gulf Coast, and northeastward to New Hampshire and Quebec; during the past century it also was transplanted into many other areas for fishing [25] (Figure 1A). After maturing at age three, it migrates annually in spring to early summer to reproduce at natal spawning grounds [35], exhibiting site fidelity, homing, and philopatry [36]. Adults do not provide parental care or nest guarding, and range widely to feed after spawning, travelling 50-300 km [37].

Past studies by our laboratory examined fine-scale population patterns of walleye using nine nuclear DNA microsatellite ( $\mu$ sat) loci, showing that most spawning groups markedly differ even within lake basins and between proximate sites, as well as at broad scales supporting natal homing and spawning site philopatry [30,38-40]. Stepien et al. [38] found that the genetic structure of spawning groups remains similar from year to year, among age cohorts, and from generation to generation. An earlier study [41] addressed patterns across the Great Lakes using mitochondrial (mt) DNA control region sequences, whose geographic scope is extended here to accompany the $\mu$ sat data set and includes new information from the Canadian Shield lakes for both data sets.

We additionally evaluate the historic genetic diversity of walleye from Lake Erie using preserved samples from 1923-1949. We address the taxonomic identity of a possible historic walleye variant, the "blue pike" S. v. "glaucus" (Hubbs [42]), whose distinction has been controversial $[41,43]$. The "blue pike" was reported to be endemic in Lakes Erie and Ontario, where it co-occurred with the common walleye $S . v$. vitreus (hereafter referred to as walleye). The "blue pike" was believed to inhabit the deeper cooler waters of eastern Lake Erie, but also was caught in the shallow and warmer western basin along with walleye [44]. It reportedly spawned somewhat later and in deeper areas than walleye [43]. The "blue pike" was described to have a steel grey-blue color, larger eyes that were higher on the head, and a smaller interorbital distance than walleye $[44,45]$. However, all characters overlapped extensively between the two [44]. Hubbs [42] originally described the "blue pike" as a separate species ("S. glaucus"), which then 


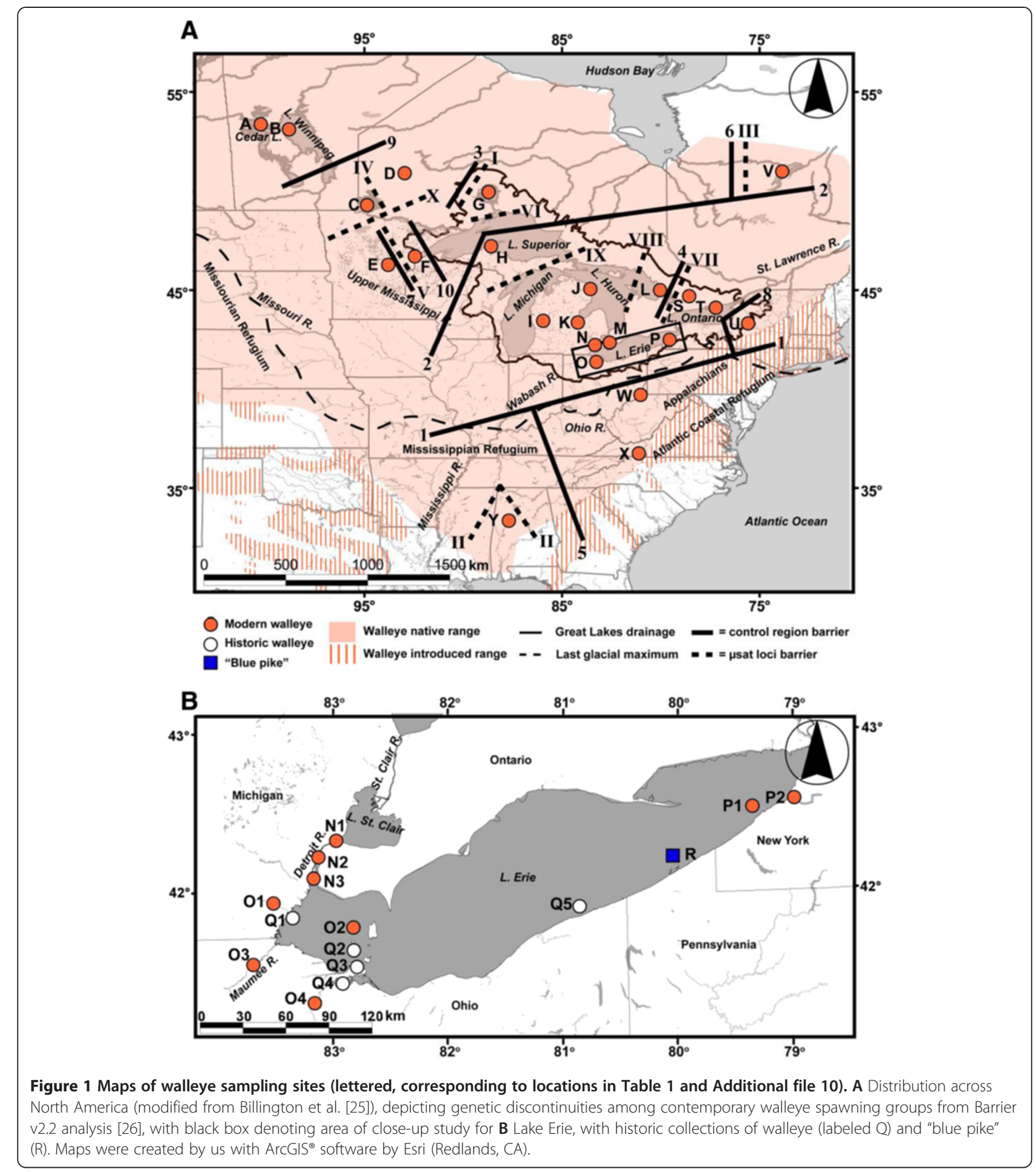

was demoted to a subspecies due to pronounced intergrades with walleye including color and all other morphological measures [44]. The present study thus addresses their identities using new morphological and genetic data.

Both "blue pike" and walleye shared a popular commercial fishery with the former collapsing in 1959 attributed to exploitation, pollution, and/or habitat alteration and the latter concurrently declining [44]. The last "blue pike" record was reported in 1965 [44] and the US Fish and Wildlife Service declared it officially extinct in 1983 [46]. The "blue pike's" identity has been confusing since it did not belong to the pike family (Esocidae) and some walleye in northern waters (along the Canadian Shield) are colored bright turquoise-blue due to sandercyanin 
protein in the mucus [47], which does not match the darker grey-blue color described for "blue pike" [48,49]. Early fishery biologists called these turquoise mucus walleye "mutants" and stated that they lacked the body color, shape, and other morphological characteristics of "blue pike" [49]; however, these variant colors have become confused in the literature [41]. Turquoise mucus and yellow walleye occur sympatrically in the same northern habitats, and the latter also have some turquoise mucus pigment [45]. The turquoise mucus additionally characterizes some yellow perch Perca flavescens (Mitchill 1814) in those habitats. Some of the turquoise color typically "rubs off" when the fish is collected [46 and CAS, pers. obs.].

This study aims to resolve population genetic patterns of walleye across its native range, providing a baseline for evaluating future anthropogenic pressures. We analyze contemporary walleye spawning groups and compare Lake Erie populations to historic samples, including the putative "blue pike" variant. Morphological characters also are analyzed for the historic samples. We expand the previous sampling coverage to 1181 walleye and combine information from mtDNA control region sequences and nine nuclear DNA $\mu$ sat loci. Patterns are evaluated at multiple evolutionary and temporal scales, with mtDNA sequences revealing historical context (glacial refugium origins and taxonomic relationships) and $\mu$ sat loci addressing contemporary microevolutionary processes (migration, gene flow, and genetic drift) [50,51]. Biogeographic patterns are compared with those of other North American taxa. Specific questions are: (1) What is the genetic structure of walleye across its native distribution?, (2) How does genetic diversity vary across the range?, (3) What is the relationship of contemporary samples to historic Lake Erie (1923-1949) patterns?, and (4) Did the extinct "blue pike" significantly differ from walleye?

\section{Results \\ Genetic diversity and phylogenetic relationships from mtDNA}

Contemporary walleye populations contain 27 control region haplotypes (Figure 2; GenBank Accession numbers U90617, JX442946-56, KC819843-54, KF954732-35). Haplotypes 1-23 match those previously described by our laboratory $[41,52,53]$ and five additional ones are identified here (four from contemporary samples, haplotypes 24-27; GenBank:KF954732-35, and one from historic Lake Erie walleye, haplotype 28; GenBank:KF954736) -totaling 28 haplotypes. Haplotype 19 from the Ohio/New Rivers $(\mathrm{W}-\mathrm{X}, 1.00 \mathrm{pp} / 98 \%)$ is located basally on the phylogenetic tree as the sister clade to all other walleye haplotypes, diverging by 19 steps (sites W and X; Additional file 1). The remaining 27 haplotypes share a common origin, with most differing by just single mutational steps, excepting haplotype 20 from the North River (site Y), which differs by eight (Figure 2A). Historic walleye haplotype 28 groups with the others (Additional file 1), varying by just a single mutational step from abundant haplotype 3 and found in a single individual.

Five abundant haplotypes $(1-4,11)$ comprise $90 \%$ of walleye individuals, with haplotype 1 being the most frequent and widely distributed (Figure 2B, Additional file 2). Haplotype 3 is the next most numerous, and is distributed from the upper Great Lakes through the south. It is the sole haplotype shared between the contemporary and historic samples; it predominated in historic Lake Erie walleye and characterized all "blue pike" samples, in contrast to being represented in just $22 \%$ of today's Lake Erie walleye (Figure 2B, Additional file 2). The historic "blue pike" samples thus do not genetically differ from historic and contemporary walleye in mtDNA control region sequences. Haplotypes 2 and 4 are less abundant, yet have wide distributions and characterize a large number of individuals and locations. Haplotype 11 is broadly distributed and abundant in the northern samples (A-G, K, V), but mostly is absent from other areas (Figure 2B, Additional file 2). Three other haplotypes appear unique to separate drainages: haplotype 25 in Lake Winnipeg and the Upper Mississippi River (A, D-E), haplotype 19 from the Ohio/New Rivers (W-X), and haplotype 20 in the North River (Y).

Each spawning group contains multiple haplotypes (Figure 2, Table 1), with Lake Erie having the most (O-P) and Lake of the Woods (C), St. Louis River (F), Moon/ Musquash Rivers (L), Lac Mistassini (V), and North River $(\mathrm{Y})$ having the least. Historic walleye and "blue pike" appear to have possessed fewer haplotypes compared to populations in Lakes Erie and Ontario today. Modest numbers and proportions of private haplotypes characterize contemporary populations, with Oneida Lake (U) and North River $(\mathrm{Y})$ containing the most. Lake Erie walleye have more private haplotypes than other Great Lakes' locations. A rare private haplotype (28) occurred in a single historic walleye individual, which appears to have been lost to today's populations. Historic "blue pike" samples lacked private haplotypes.

Haplotype diversity $\left(H_{\mathrm{D}}\right)$ varies greatly among populations (Table 1), with the Moon/Musquash Rivers (L) and Mille Lacs (E) being lowest and Ohio/New Rivers (W-X), eastern Lake Erie (P), Cedar Lake (A), and western Lake Erie $(\mathrm{O})$ highest. Historic walleye and "blue pike" samples had much lower diversity than contemporary walleye from Lakes Erie and Ontario, supported by analysis of variance (ANOVA; $F=47.24, \mathrm{df}=2, p=0.005$ ).

\section{Genetic diversity patterns from nuclear DNA loci}

All nine $\mu$ sat loci conform to Hardy Weinberg Equilibrium (HWE) expectations and are unlinked. Locus Svi17 

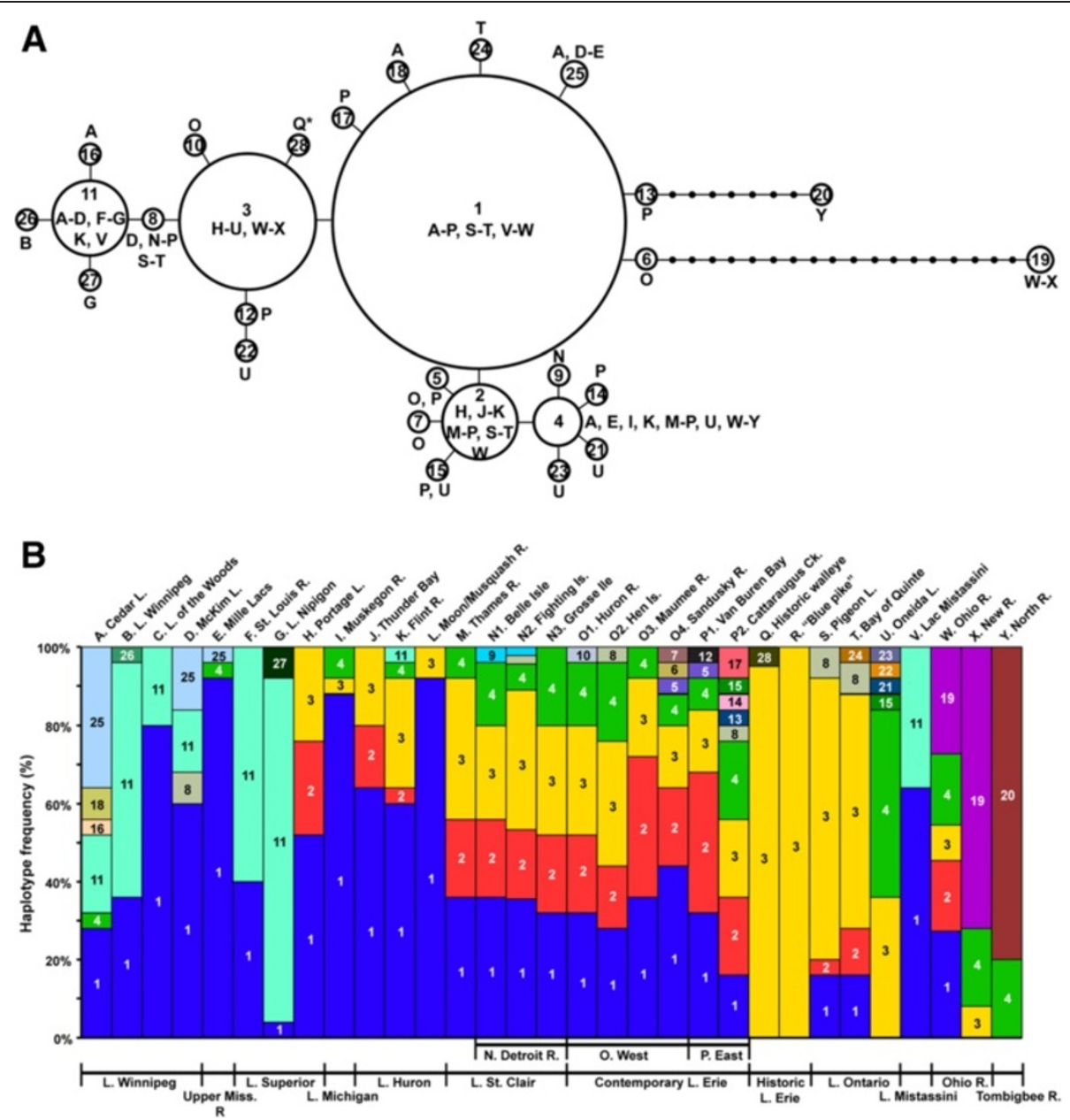

Figure 2 Relationships among mtDNA control region haplotypes of walleye, including A haplotype network from TCS v1.21 [54] and B relative frequencies per population sample. Haplotypes from contemporary spawning groups are numbered 1-27 and \#28 was found in a historic walleye. Letters = geographic locations of haplotypes from Table 1. ${ }^{*}=$ haplotypes occurring in the historic walleye and "blue pike" samples analyzed here. Circles in the network are sized according to total observed frequency of the haplotype. Lines =a single mutational step between the haplotypes; small unlabeled circles = those hypothesized/not sampled.

has a higher $F_{\mathrm{ST}}$ value, suggesting some possible positive selection, whereas SviL7 has the lowest, suggesting slight balancing selection based on results from Lositan [56] (Additional file 3). The remaining seven loci conform to neutrality, having intermediate $F_{\mathrm{ST}}$ values (mean $=0.090$ ). Locus Svi18 has the fewest alleles and Svi6 the most. Seven loci are included here for historic walleye and "blue pike", since Svi17 and L7 failed to amplify. Results from the seven versus the nine loci are identical among the 23 contemporary spawning groups, therefore, the former are presented here; the dataset based on nine loci was detailed by Stepien et al. [30,40].

Most walleye populations (87\%, 152/175 comparisons) are free of null alleles. Micro-Checker v2.2.3 [57] detects some slight homozygote excess at Svi33 (for populations C, L, V), Svi4 (C, P), Svi18 (O, Q, R, V), SviL6 (O, Q, T), Svi6 (F, Q, U, X), and Svi7 (L, M, Q). Since those loci have no such excess in other spawning groups, all populations are in HWE, and are free of heterozygote deficiency, scoring error, and stuttering [57], all loci are analyzed here. The historic walleye sample $(\mathrm{Q})$ contains some possible null alleles (57\%, Svi18, L6, 6, and 7), which appear more prevalent at loci having longer allelic lengths (Additional file 4). However, these allelic frequencies are similar among all samples: $0.00-0.20$ for contemporary walleye spawning groups, $0.02-0.22$ for historic walleye (site Q) and 0.00-0.27 for historic "blue pike" (R), negating their possible bias.

FreeNA analysis [58] results moreover discern no effect from possible null alleles on $F_{\mathrm{ST}}$ values. With FreeNA corrections, pairwise comparisons between the historic $(\mathrm{Q}-\mathrm{R})$ and contemporary samples $(\mathrm{O}-\mathrm{P}, \mathrm{S}-\mathrm{T})$ only slightly change (from 0.141 to 0.120 ), retaining a similarly large magnitude of difference between the sampling intervals. 
Table 1 Genetic variation of contemporary walleye populations (sampling areas labeled A-P, S-Y), historic walleye (Q), and "blue pike" (R) determined from mtDNA control region sequence data and nuclear DNA $\mu$ sat loci

\begin{tabular}{|c|c|c|c|c|c|c|c|c|c|c|c|}
\hline \multirow[b]{2}{*}{ Location } & \multicolumn{4}{|c|}{ Control region } & \multicolumn{7}{|c|}{ $\mu$ sat loci } \\
\hline & $\bar{N}$ & $H_{\mathrm{D}} \pm$ S.E. & $N_{\mathrm{H}}$ & $\overline{N_{\mathrm{PH}}}$ & $\bar{N}$ & $H_{\mathrm{O}} \pm$ S.E. & $F_{\mathrm{IS}}$ & $N_{\mathrm{A}}$ & $N_{\mathrm{PA}}$ & $A_{\mathrm{R}}$ & $\mathrm{Sib}$ \\
\hline \multicolumn{12}{|l|}{ L. Winnipeg } \\
\hline A. Cedar L. & 25 & $0.77 \pm 0.01$ & 6 & 2 & 25 & $0.63 \pm 0.04$ & 0.025 & 36 & 0 & 3.35 & 0.12 \\
\hline B. L. Winnipeg & 25 & $0.53 \pm 0.01$ & 3 & 1 & 25 & $0.67 \pm 0.02$ & 0.003 & 38 & 0 & 3.44 & 0.08 \\
\hline C. L. of the Woods & 25 & $0.33 \pm 0.02$ & 2 & 0 & 30 & $0.64 \pm 0.03$ & 0.099 & 39 & 0 & 3.68 & 0.00 \\
\hline D. McKim L. & 25 & $0.61 \pm 0.02$ & 4 & 0 & 25 & $0.57 \pm 0.04$ & -0.021 & 34 & 0 & 3.00 & 0.28 \\
\hline \multicolumn{12}{|l|}{ Upper Mississippi R. } \\
\hline E. Mille Lacs & 25 & $0.16 \pm 0.02$ & 3 & 0 & 39 & $0.62 \pm 0.02$ & 0.010 & 38 & 2 & 3.22 & 0.15 \\
\hline \multicolumn{12}{|l|}{ L. Superior } \\
\hline F. St. Louis R. & 25 & $0.50 \pm 0.01$ & 2 & 0 & 28 & $0.68 \pm 0.02$ & 0.116 & 56 & 0 & 4.20 & 0.14 \\
\hline G. L. Nipigon & 25 & $0.23 \pm 0.02$ & 3 & 1 & 30 & $0.74 \pm 0.03$ & -0.047 & 43 & 0 & 3.76 & 0.40 \\
\hline H. Portage L. & 25 & $0.64 \pm 0.01$ & 3 & 0 & 56 & $0.73 \pm 0.02$ & 0.001 & 52 & 0 & 3.97 & 0.07 \\
\hline \multicolumn{12}{|l|}{ L. Michigan } \\
\hline I. Muskegon R. & 25 & $0.23 \pm 0.02$ & 3 & 0 & 50 & $0.73 \pm 0.01$ & 0.057 & 57 & 0 & 4.20 & 0.04 \\
\hline \multicolumn{12}{|l|}{ L. Huron } \\
\hline J. Thunder Bay & 25 & $0.55 \pm 0.02$ & 3 & 0 & 40 & $0.70 \pm 0.01$ & 0.015 & 55 & 2 & 3.88 & 0.15 \\
\hline K. Flint R. & 25 & $0.58 \pm 0.02$ & 5 & 0 & 44 & $0.77 \pm 0.01$ & -0.023 & 55 & 0 & 4.15 & 0.09 \\
\hline L. Moon/Musquash R. & 25 & $0.15 \pm 0.02$ & 2 & 0 & 35 & $0.71 \pm 0.03$ & 0.024 & 49 & 0 & 3.87 & 0.14 \\
\hline \multicolumn{12}{|l|}{ L. St. Clair } \\
\hline M. Thames R. & 25 & $0.72 \pm 0.01$ & 4 & 0 & 39 & $0.75 \pm 0.02$ & 0.012 & 63 & 0 & 4.19 & 0.05 \\
\hline N. Detroit R. & 95 & $0.74 \pm 0.00$ & 6 & 1 & 123 & $0.71 \pm 0.01$ & 0.022 & 72 & 2 & 4.06 & 0.07 \\
\hline N1. Belle Isle & 25 & $0.78 \pm 0.01$ & 5 & 0 & 40 & $0.72 \pm 0.02$ & 0.010 & 62 & 0 & 4.06 & 0.00 \\
\hline N2. Fighting Is. & 45 & $0.73 \pm 0.01$ & 6 & 0 & 48 & $0.68 \pm 0.01$ & 0.044 & 57 & 0 & 3.92 & 0.08 \\
\hline N3. Grosse lle & 25 & $0.77 \pm 0.01$ & 4 & 0 & 35 & $0.74 \pm 0.02$ & 0.001 & 60 & 0 & 4.20 & 0.11 \\
\hline \multicolumn{12}{|l|}{ Contemporary L. Erie } \\
\hline O. Western L. Erie & 100 & $0.76 \pm 0.00$ & 9 & 3 & 211 & $0.70 \pm 0.01$ & 0.035 & 78 & 0 & 4.02 & 0.07 \\
\hline O1. Huron R. & 25 & $0.78 \pm 0.01$ & 5 & 1 & 40 & $0.73 \pm 0.02$ & 0.021 & 64 & 0 & 4.18 & 0.00 \\
\hline O2. Hen Is. & 25 & $0.78 \pm 0.01$ & 5 & 0 & 65 & $0.67 \pm 0.01$ & 0.039 & 63 & 0 & 3.84 & 0.09 \\
\hline O3. Maumee R. & 25 & $0.72 \pm 0.01$ & 4 & 0 & 76 & $0.69 \pm 0.01$ & 0.042 & 65 & 0 & 4.01 & 0.11 \\
\hline O4. Sandusky R. & 25 & $0.76 \pm 0.01$ & 7 & 2 & 30 & $0.75 \pm 0.02$ & 0.006 & 54 & 0 & 4.15 & 0.00 \\
\hline P. Eastern L. Erie & 50 & $0.82 \pm 0.00$ & 11 & 4 & 137 & $0.74 \pm 0.01$ & 0.034 & 74 & 1 & 4.30 & 0.09 \\
\hline P1. Van Buren Bay & 25 & $0.76 \pm 0.01$ & 5 & 1 & 87 & $0.76 \pm 0.01$ & 0.021 & 64 & 1 & 4.33 & 0.13 \\
\hline P2. Cattaraugus Ck. & 25 & $0.88 \pm 0.01$ & 9 & 3 & 50 & $0.71 \pm 0.02$ & 0.053 & 66 & 0 & 4.18 & 0.04 \\
\hline \multicolumn{12}{|l|}{ Historic L. Erie } \\
\hline Q. Historic yellow walleye & 20 & $0.10 \pm 0.02$ & 2 & 1 & 31 & $0.40 \pm 0.04$ & 0.258 & 44 & 6 & 2.83 & 0.23 \\
\hline R. "Blue pike" & 20 & $0.00 \pm 0.00$ & 1 & 0 & 25 & $0.54 \pm 0.07$ & -0.191 & 20 & 0 & 2.06 & 0.76 \\
\hline \multicolumn{12}{|l|}{ L. Ontario } \\
\hline S. Pigeon L. & 25 & $0.47 \pm 0.02$ & 4 & 0 & 29 & $0.73 \pm 0.02$ & -0.017 & 55 & 0 & 4.01 & 0.00 \\
\hline T. Bay of Quinte & 25 & $0.62 \pm 0.02$ & 5 & 1 & 50 & $0.69 \pm 0.02$ & 0.046 & 65 & 0 & 4.00 & 0.08 \\
\hline U. Oneida L. & 25 & $0.66 \pm 0.01$ & 6 & 3 & 25 & $0.66 \pm 0.03$ & 0.103 & 48 & 0 & 3.98 & 0.08 \\
\hline \multicolumn{12}{|l|}{ Lac Mistassini } \\
\hline V. Lac Mistassini & 25 & $0.48 \pm 0.01$ & 2 & 0 & 40 & $0.52 \pm 0.03$ & 0.137 & 45 & 2 & 3.09 & 0.05 \\
\hline
\end{tabular}


Table 1 Genetic variation of contemporary walleye populations (sampling areas labeled A-P, S-Y), historic walleye (Q), and "blue pike" (R) determined from mtDNA control region sequence data and nuclear DNA $\mu$ sat loci (Continued)

\begin{tabular}{|c|c|c|c|c|c|c|c|c|c|c|c|}
\hline \multicolumn{12}{|l|}{ Ohio R. } \\
\hline W. Ohio R. & 11 & $0.85 \pm 0.02$ & 5 & 0 & 4 & $0.61 \pm 0.07$ & 0.306 & 33 & 0 & 4.71 & 0.00 \\
\hline X. New R. & 25 & $0.45 \pm 0.05$ & 3 & 0 & 35 & $0.68 \pm 0.01$ & 0.121 & 60 & 1 & 4.29 & 0.06 \\
\hline \multicolumn{12}{|l|}{ Tombigbee R. } \\
\hline Y. North R. & 5 & $0.40 \pm 0.11$ & 2 & 1 & 5 & $0.56 \pm 0.11$ & 0.197 & 27 & 3 & 3.53 & 0.00 \\
\hline $\begin{array}{l}\text { Mean contemporary walleye } \\
(23 \text { sites; A-P, S-Y) }\end{array}$ & 31 & $0.53 \pm 0.02$ & 4 & 1 & 49 & $0.68 \pm 0.03$ & 0.055 & 39 & 1 & 3.86 & 0.08 \\
\hline
\end{tabular}

$F_{\mathrm{ST}}$ values between the contemporary populations change only at the thousandth decimal point after correction (e.g., 0.058 to 0.054 ). Thus, there was no need to adjust the allele frequencies against possible null alleles, as there was no apparent effect.

We recover 155 alleles among seven nuclear $\mu$ sat loci, across 23 contemporary walleye spawning groups, historic walleye, and "blue pike" (Table 1, Additional file 3). Many alleles are shared, widely distributed, and have high frequency in contemporary and historic samples (Additional file 4). Great Lakes' populations possess more alleles than the others, with Lake Erie housing the most, similar to control region data. Allelic richness is highest in the Ohio (W/X) and North Rivers (Y), moderate in Great Lakes' populations, and lowest in the northern sites $(\mathrm{A}-\mathrm{E}, \mathrm{V})$. ANOVA indicates that allelic richness significantly varies among populations $(F=2.50, \mathrm{df}=22$, $p<0.001)$; Tukey's post hoc tests, however, reveal no overall pattern.

Historic walleye (Q) and "blue pike" (R) possess fewer alleles and lower allelic richness (ANOVA $F=11.129$, $\mathrm{df}=5, p<0.001)$ than characterize contemporary walleye from Lakes Erie (sites $\mathrm{O}-\mathrm{P}$ ) and Ontario (S-T; Table 1, Additional file 4). Allele lengths show some tendency to be smaller in historic samples and longer in the contemporary samples (Additional file 4). Historic walleye also display some inbreeding depression, whereas "blue pike" suggest some outbreeding (Table 1), but both were in HWE. Contemporary populations comparatively have moderate values. Colony v2.0.5.0 [55] analyses indicate that full siblings constitute $8 \%$ of contemporary walleye populations, being highest in McKim Lake (D) and Lake Nipigon (G). Higher values also characterize our historic walleye and "blue pike" samples (R).

Modest proportions of private $\mu$ sat alleles typify the 23 contemporary samples, with the North River (Y) having the highest (Table 1). Historic walleye samples contained the most private alleles. However, all historic "blue pike" samples possessed common walleye alleles, with no unique ones, supporting lack of distinction.
Genetic diversity values from the $\mu$ sat loci $\left(H_{\mathrm{O}}\right)$ generally are somewhat higher than in the control region (Table 1). Diversities of historic walleye and "blue pike" were relatively low compared to contemporary samples. ANOVA indicates some differences in diversity among contemporary spawning groups $(F=1.79, \mathrm{df}=22, p=$ $0.02)$, and between contemporary versus historic samples $(F=3.83$, df $=5, p=0.007)$, however, Tukey's tests describe no overall pattern. Thus, genetic diversity is similar among all samples.

\section{Spatial genetic structure of walleye populations}

Relationships among spawning groups show a pattern of genetic isolation with geographic distance (Additional file 5). Most are genetically distinctive (Table 2, Additional file 6) for both the control region and $\mu$ sat data sets, indicating high genetic structure. The most pronounced divergences distinguish three geographic regions: northern $(\mathrm{A}-\mathrm{E})$, Great Lakes $(\mathrm{F}-\mathrm{U})$, and southeastern $(\mathrm{X}-\mathrm{Y})$. Notably, the Ohio/New (W-X) and North River (Y) populations differ by the greatest $F_{\mathrm{ST}}$ values. Geneclass2 [59] assignment tests denote high self-assignment among spawning groups and to the three geographic regions, similar to pairwise comparison results (Additional file 7). Hierarchical analysis of molecular variance (AMOVA) [60] also supports division into three geographic regions: northern (A-E, V), Great Lakes (F-U), and southeast (W-Y), which explains the most overall variation, has highest mean $F_{\mathrm{ST}}$ values, and best characterizes the data. A second well-supported scenario distinguishes walleye from each of the 11 drainage systems, with high mean $F_{\mathrm{ST}}$ values, but less support. The scenario for potential partitioning between river and lake population groups is unsupported.

Barrier v2.2 [26] and Structure v2.3.4 [61] analyses likewise identify significant genetic structuring across the range (Figures 1 and 3, Additional file 8). The first mtDNA barrier separates the southern (W-Y) from northern populations (A-P, S-V), the second isolates the northernmost sites $(A-G, V)$, and the third distinguishes Lake Nipigon 
Table 2 Genetic $F_{\mathrm{ST}}$ divergences between pairs of walleye population samples, including contemporary spawning groups (labeled A-P, S-Y), historic Lake Erie walleye (Q) and "blue pike" (R), from mtDNA control region sequences (below diagonal) and nuclear $\mu$ sat data (above diagonal)

$\begin{array}{llllllllllllllllllllllllll}\text { Location } & \text { A. } & \text { B. } & \text { C. } & \text { D. } & \text { E. } & \text { F. } & \text { G. } & \text { H. } & \text { I. } & \text { J. } & \text { K. } & \text { L. } & \text { M. } & \text { N. } & \text { O. } & \text { P. } & \text { Q. } & \text { R. } & \text { S. } & \text { T. } & \text { U. } & \text { V. } & \text { W. } & \text { X. } & \text { Y. }\end{array}$

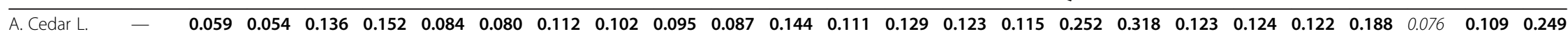
$\begin{array}{lllllllllllllllllllllllllll}\text { B. L. Winnipeg } & 0.164 & - & 0.028 & 0.150 & 0.144 & 0.080 & 0.040 & 0.111 & 0.119 & 0.144 & 0.122 & 0.155 & 0.127 & 0.141 & 0.131 & 0.113 & 0.240 & 0.281 & 0.155 & 0.143 & 0.136 & 0.189 & 0.039 & 0.128 & 0.252\end{array}$ $\begin{array}{lllllllllllllllllllllllllllll}\text { C. L. of } & 0.248 & 0.271 & - & 0.147 & 0.090 & 0.064 & 0.031 & 0.081 & 0.093 & 0.108 & 0.093 & 0.124 & 0.100 & 0.113 & 0.109 & 0.091 & 0.222 & 0.267 & 0.128 & 0.121 & 0.125 & 0.162 & 0.034 & 0.104 & 0.223\end{array}$ the Woods

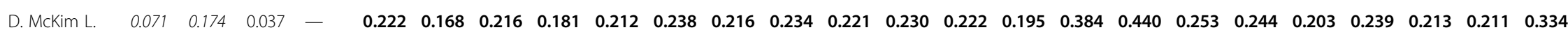
$\begin{array}{lllllllllllllllllllllllllll}\text { E. Mille Lacs } & 0.360 & 0.487 & 0.072 & 0.136 & - & 0.115 & 0.069 & 0.149 & 0.154 & 0.155 & 0.162 & 0.177 & 0.115 & 0.115 & 0.111 & 0.097 & 0.274 & 0.330 & 0.184 & 0.139 & 0.150 & 0.183 & 0.155 & 0.117 & 0.288\end{array}$ $\begin{array}{llllllllllllllllllllllllllllll}\text { F. St. Louis R. } & 0.171 & 0.000 & 0.256 & 0.167 & 0.480 & - & 0.053 & 0.035 & 0.044 & 0.055 & 0.046 & 0.061 & 0.034 & 0.045 & 0.040 & 0.029 & 0.179 & 0.211 & 0.087 & 0.067 & 0.050 & 0.138 & 0.003 & 0.044 & 0.164\end{array}$ $\begin{array}{llllllllllllllllllllllllllll}\text { G. L. Nipigon } & 0.385 & 0.173 & 0.646 & 0.501 & 0.801 & 0.203 & - & 0.086 & 0.088 & 0.094 & 0.088 & 0.119 & 0.073 & 0.082 & 0.077 & 0.069 & 0.190 & 0.242 & 0.110 & 0.093 & 0.108 & 0.168 & 0.031 & 0.085 & 0.215\end{array}$ $\begin{array}{lllllllllllllllllllllllllllll}\text { H. Portage L. } & 0.173 & 0.280 & 0.167 & 0.094 & 0.236 & 0.280 & 0.557 & - & 0.044 & 0.066 & 0.047 & 0.036 & 0.043 & 0.055 & 0.054 & 0.044 & 0.169 & 0.200 & 0.090 & 0.074 & 0.044 & 0.130 & 0.014 & 0.051 & 0.212\end{array}$ $\begin{array}{lllllllllllllllllllllllllllllll}\text { I. Muskegon R. } & 0.334 & 0.446 & 0.054 & 0.117 & 0.000 & 0.439 & 0.765 & 0.187 & - & 0.028 & 0.007 & 0.052 & 0.031 & 0.046 & 0.042 & 0.041 & 0.095 & 0.135 & 0.047 & 0.041 & 0.042 & 0.167 & 0.008 & 0.031 & 0.156\end{array}$ $\begin{array}{lllllllllllllllllllllllllllll}\text { J. Thunder } & 0.196 & 0.301 & 0.098 & 0.064 & 0.145 & 0.297 & 0.603 & 0.000 & 0.098 & - & 0.011 & 0.073 & 0.027 & 0.036 & 0.035 & 0.040 & 0.119 & 0.184 & 0.036 & 0.031 & 0.066 & 0.196 & 0.035 & 0.042 & 0.188\end{array}$ Bay

K. Flint R.

L. Moon/

Musquash R.

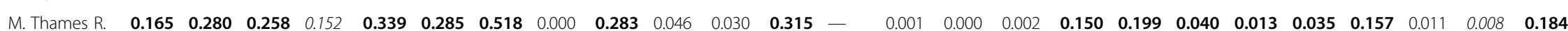
$\begin{array}{lllllllllllllllllllllllllllllllll}\text { N. Detroit R. } & 0.158 & 0.253 & 0.213 & 0.138 & 0.259 & 0.255 & 0.437 & 0.011 & 0.221 & 0.053 & 0.041 & 0.243 & 0.000 & - & 0.001 & 0.007 & 0.142 & 0.191 & 0.045 & 0.020 & 0.058 & 0.166 & 0.035 & 0.021 & 0.198\end{array}$ $\begin{array}{llllllllllllllllllllllllllllll}\text { O. Western } & 0.148 & 0.242 & 0.200 & 0.126 & 0.244 & 0.243 & 0.426 & 0.007 & 0.208 & 0.048 & 0.049 & 0.233 & 0.000 & 0.000 & - & 0.006 & 0.136 & 0.182 & 0.048 & 0.017 & 0.048 & 0.160 & 0.024 & 0.018 & 0.198\end{array}$ L. Erie

P. Eastern

L. Erie

Q. Historic

walleye

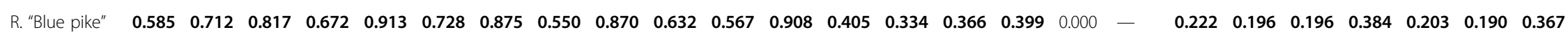
$\begin{array}{llllllllllllllllllllllllllll}\text { S. Pigeon L. } & 0.351 & 0.471 & 0.541 & 0.402 & 0.635 & 0.484 & 0.651 & 0.247 & 0.583 & 0.322 & 0.253 & 0.610 & 0.119 & 0.136 & 0.171 & 0.194 & 0.092 & 0.145 & - & 0.010 & 0.082 & 0.225 & 0.031 & 0.055 & 0.196\end{array}$ $\begin{array}{lllllllllllllllllllllllllllll}\text { T. Bay of } & 0.272 & 0.392 & 0.455 & 0.319 & 0.547 & 0.403 & 0.576 & 0.155 & 0.495 & 0.233 & 0.182 & \mathbf{0 . 5 2 2} & 0.046 & 0.073 & 0.102 & 0.112 & 0.151 & 0.205 & 0.000 & - & 0.056 & 0.198 & 0.030 & 0.032 & 0.198\end{array}$ Quinte

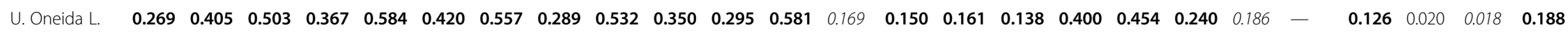
$\begin{array}{lllllllllllllllllllllllllllll}\text { V. L. Mistassini } & 0.163 & 0.088 & 0.022 & 0.027 & 0.226 & 0.072 & 0.463 & 0.161 & 0.191 & 0.131 & 0.119 & 0.230 & 0.218 & 0.191 & 0.179 & 0.211 & 0.694 & 0.739 & 0.473 & 0.389 & 0.430 & - & 0.177 & 0.136 & 0.324\end{array}$ $\begin{array}{llllllllllllllllllllllllllllllll}\text { W. Ohio R. } & 0.116 & 0.259 & 0.293 & 0.142 & \mathbf{0 . 4 1 3} & 0.269 & \mathbf{0 . 5 3 1} & 0.068 & 0.346 & 0.118 & 0.116 & \mathbf{0 . 4 1 6} & 0.041 & 0.034 & 0.020 & 0.004 & 0.557 & \mathbf{0 . 6 2 9} & \mathbf{0 . 2 8 7} & 0.180 & 0.151 & 0.222 & - & 0.010 & 0.122\end{array}$ $\begin{array}{llllllllllllllllllllllllll}\text { X. New R. } & 0.382 & 0.508 & 0.607 & 0.470 & 0.693 & 0.523 & 0.660 & 0.443 & 0.653 & 0.492 & 0.467 & 0.695 & 0.384 & 0.338 & 0.331 & 0.310 & 0.685 & 0.732 & 0.512 & 0.438 & 0.364 & 0.533 & 0.169 & - & 0.171\end{array}$ $\begin{array}{lllllllllllllllllllllllllllll}\text { Y. North R. } & 0.342 & 0.506 & 0.651 & 0.454 & 0.793 & 0.527 & 0.736 & 0.432 & 0.732 & 0.495 & 0.468 & 0.798 & 0.369 & 0.338 & 0.327 & 0.288 & 0.836 & 0.923 & 0.551 & 0.447 & 0.361 & 0.541 & 0.299 & 0.543 & - & -\end{array}$ 

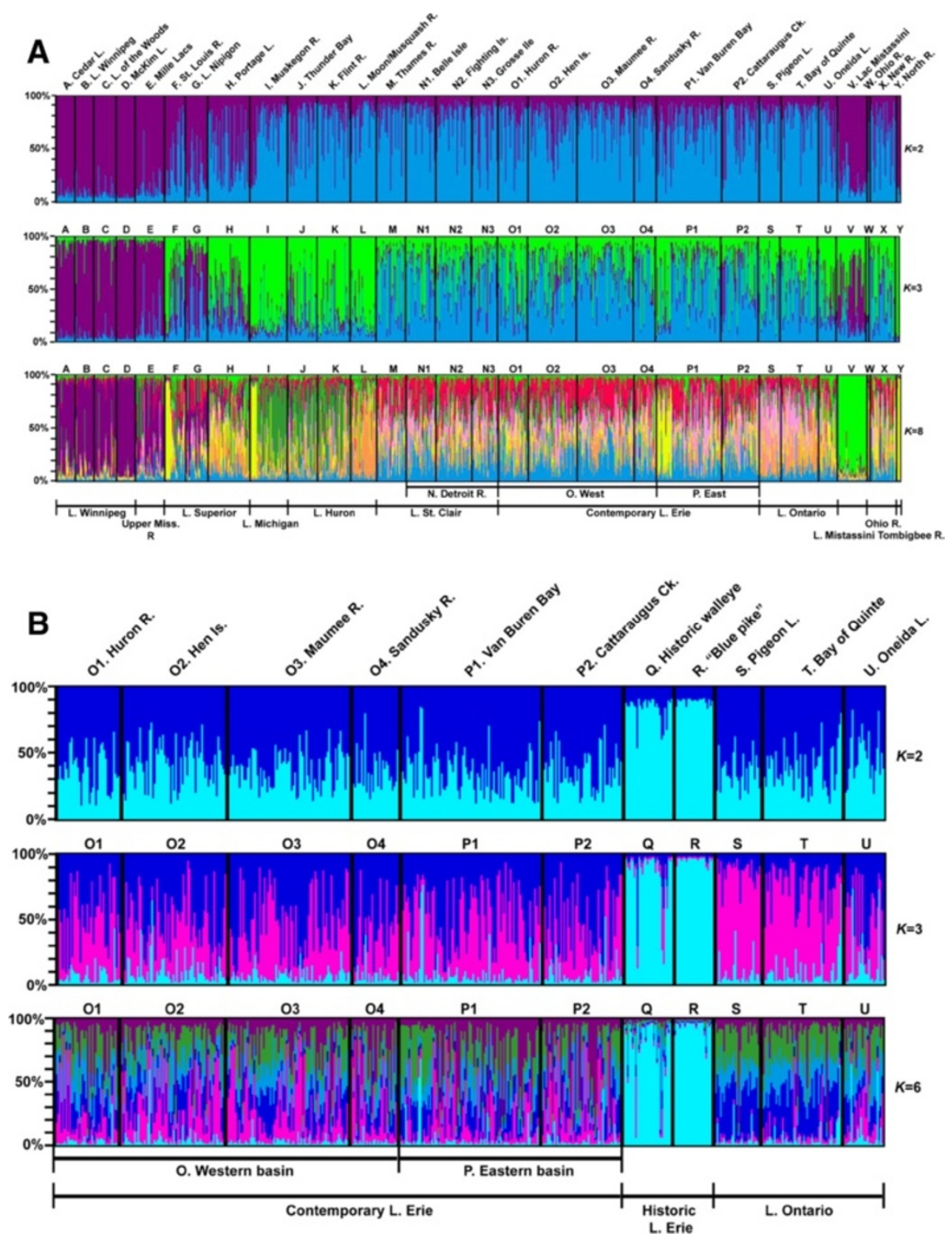

Figure 3 Bayesian Structure analysis [61] for A contemporary walleye $(K=2,3,8)$ and B contemporary Lakes Erie and Ontario and historic Lake Erie walleye (with "blue pike"; $K=2,3,6$ ) population groups, based on seven $\mu$ sat loci. Individuals are represented as thin vertical lines, partitioned into $K$ colored segments.

(G). In contrast, barriers for $\mu$ sat loci denote finer-scale discontinuities, with the first separating Lake Nipigon (G, barrier I; 49\% bootstrap support, 7/7 loci), then the North River (Y, II; 57\%, 7/7), followed by Lac Mistassini (V, III; 43\%, 6/7). Remaining barriers for both data sets depict fine-scale divergences, with most among northern spawning groups (Figure 1). Structure analyses indicate high assignment of the northernmost populations (A-G, V, purple color) from those to the south (I-U, W-Y, blue; Figure 3A). Populations in the upper (F-L; orange) and lower (M-U; light blue) Great Lakes assign separately, with St. Louis River (F) and Lake Nipigon (G) walleye clustering more closely to those in the northwest. Finerscale demarcations by drainage and some spawning groups also are recovered, similar to results from the analyses using pairwise comparisons, Geneclass, and AMOVA.

The combined population genetic distance tree (Additional file 9) also shows separation of the southern samples, with the North River ( $\mathrm{Y}$ ) walleye as basal, matching its high pairwise divergence (Table 2). The tree clusters northern walleye and those from Lake Superior together 
(sites A-G, V; 62\%), similar to Barrier results. Lakes St. Clair $(\mathrm{M}-\mathrm{N})$ and Erie populations $(\mathrm{O}-\mathrm{P})$ display close genetic relationship, analogous with pairwise comparisons.

\section{Historic vs. contemporary populations of Lake Erie walleye}

Historic walleye and "blue pike" samples are indistinguishable in control region sequences, and vary from each other in $\mu$ sat allele frequencies within the realm among contemporary walleye populations, on the low end among the 23 spawning groups (Table 2, Additional file 6). In contrast, greater values distinguish walleye populations spawning in the New (X) and North Rivers (Y). Thus, there is no genetic evidence that the "blue pike" comprised a distinct taxon from walleye.

Both sets of historic samples differed in haplotypic and allelic frequencies from contemporary Lakes Erie and Ontario walleye in the $F_{\mathrm{ST}}$ and $\chi^{2}$ analyses. The historic samples significantly varied in genetic composition from all but one contemporary spawning group (Table 2, Additional file 6). The control region frequencies of the Pigeon Lake spawning group (S) alone resemble the historic samples from Lake Erie due to shared high representation of haplotype 3 , but differ in the $\mu$ sat data. This likely is an artifact.

No genetic distinction occurs between turquoisecolored mucus versus yellow walleye from McKim Lake (D, control region: $F_{\mathrm{ST}}=0.000, \chi^{2}=0.00$, $\mu$ sat: $\left.F_{\mathrm{ST}}=0.000, \chi^{2}=14.05\right)$. Together, McKim Lake represents a distinct spawning group, differing from other walleye populations (Table 2, Additional file 6). The historic walleye and "blue pike" samples from Lake Erie were very significantly different from the McKim Lake samples. Thus, both turquoise mucus walleye and "blue pike" are walleye.

AMOVA analyses show no significant difference between the historic (walleye and "blue pike" considered together as a single group) versus the contemporary samples for control region sequences. The historic and contemporary samples differ with $\mu$ sat data (Table 3 ) by $\sim 2-5 x$ greater than the mean $F_{\mathrm{ST}}$ values among contemporary walleye spawning groups. Results thus indicate that $\mu$ sat allelic frequencies have changed from the past to the present.

The genetic distance tree of the combined gene data (Additional file 9) and Structure analyses (Figure 3B, Additional file 8) both separate the historic samples into a distinct group. Geneclass assignment tests likewise depict their genetic difference, with $89 \%$ of the historic samples self-assigning. Just $10 \%$ mis-assign to the contemporary eastern Lake Erie walleye (group P), 6\% to historic "blue pike" (R) and 6\% to contemporary western Lake Erie walleye $(\mathrm{O})$. Historic "blue pike" individuals selfassigned $48 \%$ of the time $(N=12)$, with $48 \%$ mis-assigning to historic walleye. A single "blue pike" individual assigned to contemporary eastern Lake Erie walleye (4\%).

\section{Morphological traits of historic samples}

Morphological variations occur between walleye and "blue pike" in six characters; "blue pike" individuals tend to have the smallest head and interorbital widths, largest eye diameter, highest numbers of pelvic fin rays, a larger orbit: interorbital ratio, and fewer second dorsal fin rays (Table 4). However, the ranges of all characters

Table 3 Relative distribution of genetic variation among contemporary and historic walleye samples using AMOVA, calculated from mtDNA control region sequence and nuclear DNA $\mu$ sat data

\begin{tabular}{|c|c|c|c|c|c|c|}
\hline \multirow[b]{2}{*}{ Source of variation } & \multicolumn{3}{|c|}{ Control region } & \multicolumn{3}{|c|}{ Microsatellite loci } \\
\hline & \% variation & $\Phi$ value & Mean $F_{\mathrm{ST}}$ & $\%$ variation & $\Phi$ value & Mean $F_{\mathrm{ST}}$ \\
\hline 1. Contemporary $(\mathrm{O}-\mathrm{P}, \mathrm{S}-\mathrm{T})$ vs. historic samples $(\mathrm{Q}-\mathrm{R})$ & 26.01 & $0.260 \mathrm{NS}$ & 0.260 & 6.99 & $0.070 \mathrm{NS}$ & 0.167 \\
\hline Among sampling sites within groups & 0.00 & $0.000 \mathrm{NS}$ & 0.176 & 0.87 & $0.009^{* *}$ & 0.030 \\
\hline Within sampling sites & 73.98 & $0.252^{* *}$ & - & 92.14 & $0.079^{* *}$ & - \\
\hline $\begin{array}{l}\text { 2. Among northern }(A-E, V), \text { Great Lakes } \\
(F-U) \text {, and southeast }(W-Y) \text { regions }\end{array}$ & 12.06 & $0.121^{* *}$ & 0.318 & 5.07 & $0.051^{* *}$ & 0.129 \\
\hline Among sampling sites within groups & 17.64 & $0.201^{* *}$ & 0.238 & 4.97 & $0.052^{* *}$ & 0.063 \\
\hline Within sampling sites & 70.30 & $0.297^{* *}$ & - & 89.96 & $0.100^{* *}$ & - \\
\hline 3. Among the contemporary 11 drainages $(A-P, S-Y)$ & 14.38 & $0.144^{* *}$ & 0.286 & 4.26 & $0.043^{* *}$ & 0.132 \\
\hline Among sampling sites within drainages & 11.07 & $0.129^{* *}$ & 0.158 & 3.13 & $0.033^{* *}$ & 0.058 \\
\hline Within the sampling sites & 74.05 & $0.254^{* *}$ & - & 92.61 & $0.074^{* *}$ & - \\
\hline $\begin{array}{l}\text { 4. Between lake }(A-E, G-H, J, O 2, P 1, S-V) \text { and river } \\
(F, I, K-O 1, O 3-4, P 2, W-Y) \text { spawners }\end{array}$ & 0.00 & $0.000 \mathrm{NS}$ & 0.306 & 0.58 & $0.006^{* *}$ & 0.107 \\
\hline Among sampling sites within groups & 24.92 & $0.247^{* *}$ & 0.298 & 6.59 & $0.066^{* *}$ & 0.100 \\
\hline Within sampling sites & 75.08 & $0.241^{* *}$ & - & 92.83 & $0.071^{* *}$ & - \\
\hline
\end{tabular}

NS = not significant, ${ }^{* *}=$ significant. 
Table 4 Morphological data from historic walleye and "blue pike" samples, with mean, standard deviation (SD), and range of values

\begin{tabular}{|c|c|c|c|c|}
\hline \multirow[b]{2}{*}{ Measurement } & \multicolumn{2}{|c|}{ Historic walleye $(N=51)$} & \multicolumn{2}{|c|}{ "Blue pike" $(N=52)$} \\
\hline & Mean \pm SD & Range & Mean \pm SD & Range \\
\hline Standard length (mm) & $264.3 \pm 49.9$ & $187-385$ & $248.8 \pm 37.7$ & $202-362$ \\
\hline \multicolumn{5}{|l|}{$\%$ Standard length } \\
\hline Body depth & $20.1 \pm 1.9$ & $15.8-24.2$ & $20.4 \pm 2.1$ & $16.3-24.9$ \\
\hline Head length & $30.7 \pm 0.8$ & $28.9-32.2$ & $30.6 \pm 1.8$ & $27.9-40.7$ \\
\hline \multicolumn{5}{|l|}{$\%$ Head length } \\
\hline Cheek length & $48.3 \pm 1.8$ & $44.3-52.5$ & $48.4 \pm 1.6$ & $46.3-55.7$ \\
\hline Upper jaw length & $38.6 \pm 3.1$ & $33.6-43.9$ & $37.9 \pm 3.7$ & $31.0-44.9$ \\
\hline Lower jaw length & $38.8 \pm 4.1$ & $30.1-43.7$ & $38.2 \pm 4.3$ & $31.6-44.1$ \\
\hline Head width & $41.6 \pm 4.5$ & $34.3-55.0$ & $38.6 \pm 2.5$ & $32.1-44.0^{* *}$ \\
\hline Interorbital width & $14.9 \pm 1.1$ & $12.8-17.3$ & $14.1 \pm 1.2$ & $11.2-17.2^{* *}$ \\
\hline Orbit diameter & $20.7 \pm 1.8$ & $17.4-26.4$ & $21.9 \pm 2.1$ & $16.6-25.9^{* *}$ \\
\hline Orbit: interorbital & $1.4 \pm 0.2$ & $1.1-2.0$ & $1.6 \pm 0.2$ & $1.0-2.2^{* *}$ \\
\hline Upper: lower jaw & $1.0 \pm 0.2$ & $0.8-1.3$ & $1.0 \pm 0.2$ & $0.7-1.3$ \\
\hline Meristic & Mode & Range & Mode & Range \\
\hline First dorsal fin rays & 14 & $11-16$ & 12 & $11-15$ \\
\hline Second dorsal fin rays & 21 & $19-22$ & 20 & $13-22^{* *}$ \\
\hline Pectoral fin rays & 15 & $11-17$ & 14 & $10-16$ \\
\hline Pelvic fin rays & 6 & $5-8$ & 7 & $5-8^{* *}$ \\
\hline Anal fin rays & 14 & $11-16$ & 14 & $11-16$ \\
\hline
\end{tabular}

Body and head measurements are given as percentage of standard or head lengths, respectively. Ratios are between the original measurement values.

Additional file 11 lists individuals examined. ${ }^{* *}=$ statistically significant comparisons between walleye and "blue pike" using Student's $t$ and Mann Whitney $U$ tests. Overall, walleye and "blue pike"differed according to the nonparametric MANOVA (morphometric: $F=4.34$, degrees of freedom (df) $=1, p<0.001 ;$ meristic: $F=4.13$, $\mathrm{df}=1, p=0.001)$

extensively overlap and do not reliably distinguish a "blue pike" from a walleye specimen.

The first four morphometric principal components (PC) explain $86 \%$ of the overall variation and the first three meristic PCs explain 79\% between historic walleye and "blue pike". Multivariate analysis of variance (MANOVA) shows significant difference in morphometric (Wilks' $\Lambda=0.768$, $F=7.244, \mathrm{df}=1, p<0.001)$ and meristic principal components analysis (PCA) (Wilks' $\Lambda=0.876, F=4.522, \mathrm{df}=1$, $p=0.005$; Figure 4). Morphometric PC3 and 4 distinguish walleye and "blue pike" (ANOVA, PC3: $F=10.443$, $\mathrm{df}=1, p=0.002$, PC4: $F=9.004, \mathrm{df}=1, p=0.003)$ and are correlated with body depth $(r=0.850)$ and head width $(r=-0.546)$. Meristic PC1 and 2 (Figure 4$)$ also differentiate them (ANOVA, PC1: $F=4.323, \mathrm{df}=1, p=0.040$, PC2: $F=5.0832, \mathrm{df}=1, p=0.026)$ in number of second $(r=-0.827)$ and anal fin rays $(r=-0.578)$.

Quadratic discriminant function analysis (DFA) correctly assigns $72 \%$ of samples using the morphometric dataset and just $64 \%$ with meristic characters. Historic walleye $(76 \%$ morphometric, $71 \%$ meristic data) and "blue pike" (68\%, 57\%) more often self-assign, but have high mis-assignment to each other, similar to the Geneclass results. Thus our findings do not support designation of "blue pike" as a subspecies or species, since it lacks reliable morphological characters and has no genetic differentiation, with no unique haplotypes or alleles. Its level of frequency difference in $\mu$ sat allelic composition appears typical of a walleye population.

\section{Discussion}

\section{Broadscale genetic divergence patterns}

Walleye populations from the unglaciated southern portion of the range (W-Y) are the most divergent, possessing unique alleles and haplotypes. The earliest and most divergent haplotype (19) occurs in the Ohio and New Rivers $(\mathrm{W}-\mathrm{X})$, and is very genetically distinct $([30,62]$ this study). Other fishes, including brook trout Salvelinus fontinalis (Mitchill 1814) [63], smallmouth bass Micropterus dolomieu Lacepède 1802 [64], greenside darter Etheostoma blennioides Rafinesque 1819 [65], rainbow darter Etheostoma caeruleum Storer 1845 [66], and yellow perch [67], likewise demonstrate pronounced population divergence of Atlantic coastal groups, indicating long-term isolation.

The North River (Y), which drains south into the Gulf of Mexico, is dominated by an endemic walleye haplotype (20). The population is small, very isolated, and relatively 

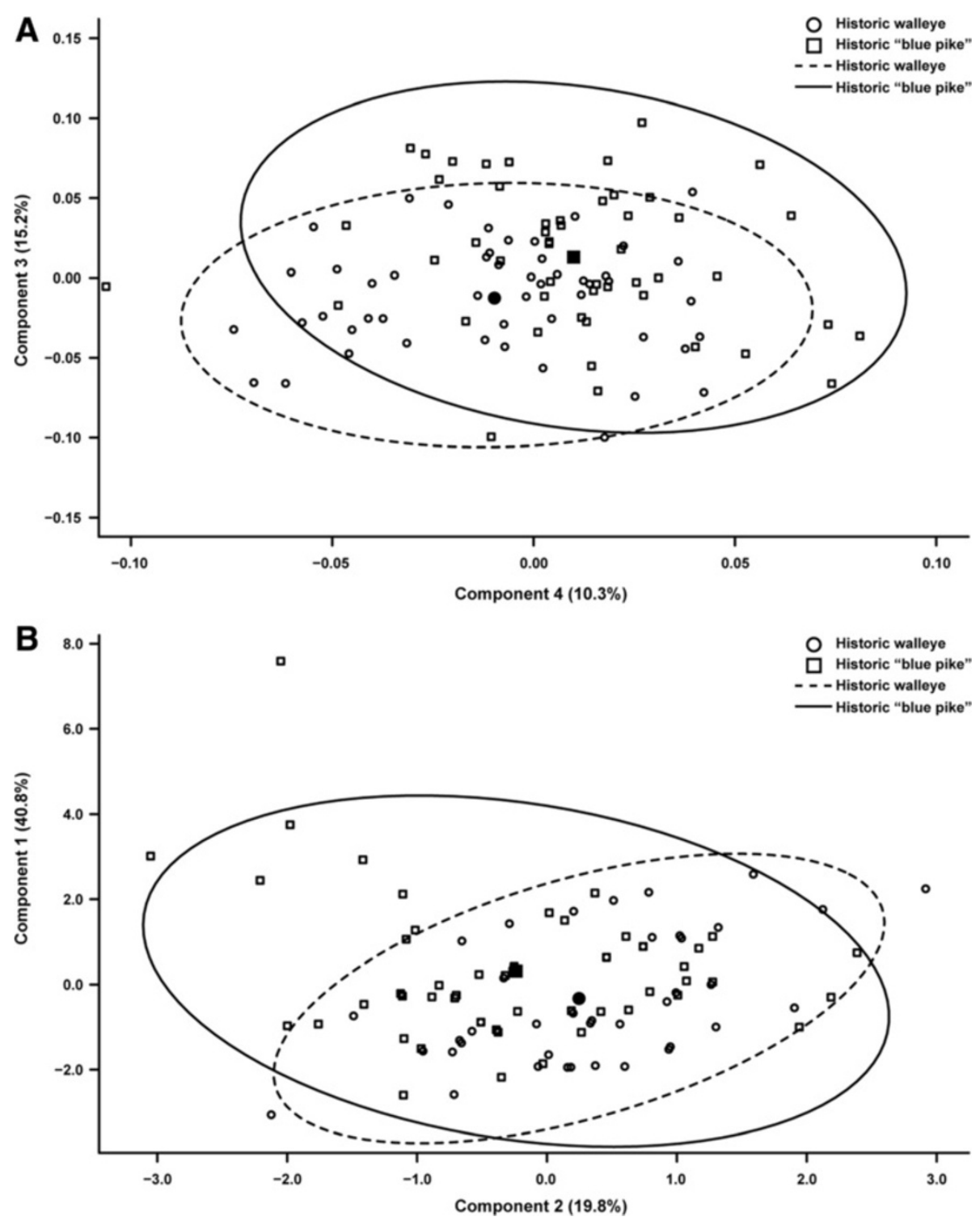

Figure 4 Principal components analysis plots for historic walleye and "blue pike", based on A morphometric and B meristic characters. Solid symbols $=$ mean component scores. Circles $=95 \%$ confidence intervals for each group.

low in diversity, as previously documented [25,30]. Boschung and Mayden [68] noted that this indigenous North River population is at risk of colonization from northerly walleye due to connections through the Tombigbee-Tennessee River waterway, which may threaten localized adaptations. Likewise, yellow perch from the Gulf Coastal drainage possessed high endemism, unique haplotypes, and extensive divergence [67]. Southerly populations of walleye and yellow perch thus represent unique genetic sources that may prove valuable for conservation.

Walleye populations exhibit moderate divergence levels across the range, as described by previous studies $[30,41,69]$. In contrast, yellow perch (control region mean
$F_{\mathrm{ST}}=0.469$, $\mu$ sat $\left.=0.236\right)[67]$ and smallmouth bass (cytochrome $b$ mean $F_{\mathrm{ST}}=0.412, \mu$ sat $\left.=0.232\right)[64,70]$ possess much higher divergence among spawning groups. This may be due to their more limited migration [71,72]. Walleye have been documented to disperse $50-300 \mathrm{~km}$ [73], followed by yellow perch to $48 \mathrm{~km}$, with occasional individuals travelling $200 \mathrm{~km}$ [71], and smallmouth bass only to $\sim 10 \mathrm{~km}[72]$.

The European perch P. fluviatilis Linnaeus 1758 discriminates kin from non-kin via olfactory cues and schools in family groups, which may reproduce together $[74,75]$. This life history pattern remains to be tested for yellow perch, walleye, or smallmouth bass. Kinship tests by our laboratory reveal high proportions of full siblings 
in some spawning groups of yellow perch (mean $=0.20$ ranging to $75 \%$ ) [76] and smallmouth bass ( 0.15 , to $67 \%)$ [70], which are greater than those identified here for walleye. Limited lifetime migration, and apparent close association among kin for yellow perch and smallmouth bass, may lead to their higher divergences among proximate populations. Walleye populations display intermediate divergence and higher diversity, attributable to more outbreeding. Thus, biogeographic patterns of these widely distributed freshwater fishes largely result from a combination of extrinsic (i.e., changes in climate and drainage patterns) and intrinsic factors (dispersal capabilities, degree of natal homing, population size, and inbreeding), which are regulated by behavior of the species.

\section{Contemporary genetic diversity patterns}

Our data indicate relatively high genetic diversity for most walleye spawning groups; these values mirror those reported across their range using nine $\mu$ sat loci $\left(H_{\mathrm{O}}=0.68\right)$ [30]. Difference between the mtDNA and nuclear data is attributable to the former having a slower evolutionary rate [51,77], $1 / 4$ effective the population size, and being more influenced by population bottlenecks [50].

Walleye reproducing in the lower Great Lakes (Lakes St. Clair and Erie) have the highest diversity values, reflecting admixture from glacial refugia and larger population sizes, similar to results from other studies [30,40]. Some small upper Great Lakes populations display the lowest mtDNA diversities, but average nuclear DNA diversities. Notably, the population from the Moon/Musquash River (site L) has the least control region diversity but higher $\mu$ sat value. Gatt et al. [78] also recovered low mtDNA diversity at this location, attributing this to stocking and overexploitation. Since this pattern is restricted to mtDNA alone in our study, it likely instead reflects past bottlenecking and small population size.

Yellow perch populations possess much lower mtDNA control region diversity levels across their range (mean $\left.H_{\mathrm{D}}=0.31\right)$ than nuclear DNA variability $\left(H_{\mathrm{O}}=0.53,15\right.$ $\mu$ sat loci) [67]. This also is true for the related European perch [79] and Eurasian ruffe Gymnocephalus cernua (Linnaeus 1758) [80]. This difference among percid species may reflect their respective evolutionary history and behavior. Notably, strong association of European perch in kin groups [74,75] and high proportions of yellow perch full siblings in spawning groups [76] may lead to lower diversity from inbreeding. Smallmouth bass spawning groups also have lower genetic diversity than walleye in mtDNA sequences (mean $H_{\mathrm{D}}=0.50$ ) [70] and eight $\mu$ sat loci $\left(H_{\mathrm{O}}=0.46\right)$ [64]. This may reflect higher association of kin groups and limited lifetime migration [70]. Thus, population genetic diversity and divergence of smallmouth bass [64,70] and yellow perch [76] differ from walleye due to their respective reproductive behavior and life history characters.

Small isolated southern populations of walleye have lower genetic diversity values than those in once glaciated regions. The former contain the oldest and most unique genetic variants, reflecting long-term isolation, smaller population sizes, and likely genetic drift and population bottlenecks [30]. Gulf Coastal yellow perch populations also possess the lowest diversity values, along with unique haplotypes and alleles [67], similar to walleye. These southern populations comprise a historic source of diversity and an important genetic resource for both species. Their adaptations to warmer habitats may provide a critical genetic reservoir in the face of climate warming.

\section{Genetic patterns shaped by anthropogenic factors in Lake Erie}

Genetic diversity values for mtDNA and $\mu$ sat loci are 94\% and 37\% lower for historic Lake Erie walleye and "blue pike" from 1923-49 compared with contemporary Lakes Erie and Ontario populations. Genetic diversity appears to have increased over the past $70+$ years, which may reflect population recovery.

Europeans settled Lake Erie shores during the 17001800 s, cutting down the forests and draining the marshlands, which disappeared by 1900 [44,81]. As the region developed and industrialized, untreated wastes were released into the Lake from saw mills, slaughterhouses, and steel factories. By 1830, Lake Erie walleye comprised an abundant and important commercial fishery. In 1874, construction of a major shipping channel drastically modified the Lakes Huron-Erie Corridor connection (Figure 1). Lake Erie steadily lost much of its fish habitat from 1900-1970s due to draining of wetlands, armoring of shorelines, channelization, dredging, and increased industrialization [82,83]. During the 1960s, high levels of phosphorus caused massive algal blooms, accompanied by oxygen depletion and marked fish die offs [82].

Many native Lake Erie fish populations experienced steady declines from 1900-1970s, including the lake trout, lake sturgeon Acipenser fulvescens Rafinesque 1817, and walleye $[81,84]$. Industrial outputs resulted in heavy metal contamination and declining fish health, manifested with neoplasms, tumors, and lesions on walleye and other species [85]. In 1970, walleye fisheries from Lakes Huron through Erie were closed due to high mercury levels. By 1978, fisheries managers declared Lake Erie walleye as being in crisis from overfishing and pollution [82].

Lower allelic numbers and diversity for historic walleye and "blue pike" from 1923-49 likely reflect these population declines. The rare haplotypes and alleles we identify in historic walleye may have disappeared. Most haplotypes and almost all alleles in historic Lake Erie samples are common and widespread today. The historic samples 
appeared to possess more haplotypes that trace to the Atlantic Refugium, whereas those from the Mississippian Refugium dominate today's walleye populations [21,25,41]. This change merits further testing, but may be a response to climate warming.

Genetic diversity of other Great Lakes fishes similarly was lower in 1927-59 and higher in 1995-2005, attributed to population declines from environmental conditions and overexploitation [86,87]. Notably, lake whitefish Coregonus clupeaformis (Mitchill 1818) from Lakes Huron and Erie in 1927 had lower diversity at seven $\mu$ sat loci (0.60) than in 1997-2005 (0.65) [87]. Lake trout from Lakes Superior, Michigan, and Huron were less variable in 1940-59 (0.47) than in 1995-99 (0.51) using five $\mu$ sat loci [86]. Similarly, Atlantic cod Gadus morhua Linnaeus 1758 declined in diversity and number of alleles from 1954-80s, then increased from 1980-98 according to archived otolith samples and three $\mu$ sat loci [88]. That study moreover documented that genetic composition changed, similar to the pattern here for Lake Erie walleye, hypothesizing recovered diversity via immigration from a nearby spawning group.

Contemporary Lake Erie walleye populations also may have been influenced by migration and recruitment. Walleye is described to natally home, with chemical cues presumably facilitating recognition of reproductive grounds [89,90]. Olson and Scidmore [89] discerned lower homing in areas with high habitat degradation. Some contemporary western Lake Erie spawning group samples appear genetically similar to those from Lake St. Clair, suggesting possible genetic exchange. Walleye movement between these lakes during non-spawning times is well known from tagging [91,92] and Lake Erie walleye may have migrated to spawn at a recently augmented reef in the Detroit River [53].

Lake Erie walleye appear to have recovered from declines in diversity and numbers ( 10 million in 1978), after the fishery's closure from 1970-76 [93], implementation of the 1970 Canada Water Act, the 1972 U.S. Clean Water Act, and the 1972 Canada-U.S. Great Lakes Water Quality Agreement [82]. Increasing water temperature -especially in the shallow western basin- changed the Lake Erie fish community from cold water (e.g., lake trout) to warmer water species, favoring walleye and yellow perch. Declining numbers of colder water competitors presumably enhanced walleye abundance [84]. By 1988, Lake Erie had rebounded to $\sim 80$ million ( $8 \mathrm{x}$ 1978), decreasing to 18 million walleye in 2013 [94]. Genetic analyses [38] revealed temporal consistency in genetic diversity from 1995-2008 for three primary Lake Erie walleye spawning groups (Maumee and Sandusky Rivers in the west, and Van Buren Bay in the east). Whether this continues remains to be discerned, as genetic variability may decline with population sizes.
Other walleye populations have shown a mixture of temporal stability and decline. Walleye spawning in Escanaba Lake, Wisconsin displayed consistent diversity levels from 1952-2002 (mean $H_{\mathrm{O}}=0.76$ ) using eight $\mu$ sat loci [95] (six of those here). Garner et al. [96] likewise described consistent diversity levels for walleye in Lake Superior's Black Bay from 1966-2010 (mean $H_{\mathrm{O}}=$ 0.62) employing nine $\mu$ sat loci (six of those here). However, the Escanaba Lake and Black Bay populations were stocked, likely circumventing fluctuations. MtDNA restriction fragment length polymorphism diversity of walleye spawning in Lake Huron's Georgian Bay declined over three decades ( 0.50 in the 1960 s to 0.15 in the 1990s), attributed to exploitation and stocking [78]. Our study recovers similarly low mtDNA control region variability for walleye spawning in the Moon/Musquash Rivers of Georgian Bay today. However, this bottleneck effect is restricted to mtDNA, since we denote average levels of nuclear DNA variability.

Other alternatives may explain lower diversity levels in the historic samples. Our contemporary samples were adults collected from spring spawning runs at specific spawning sites. In contrast, historic samples were collected from July-November, when walleye intermix. Thus genetic diversity may have been lowered due to population admixture via a Wahlund effect [97]. For historically archived samples, such as ours, Nielsen and Hansen [98] recommended including positive and negative controls, having a separate laboratory space and separate chemicals, testing for null alleles with MicroChecker, using samples with complete documentation of biological information, testing for HWE, and applying more than one statistical test to validate patterns. We followed all of these precautions to ensure reliability of data from our formalin fixed historic samples. Some studies have documented issues with historic samples having biased amplification of shorter length alleles $[99,100]$. We found slight suggestion of null alleles in historic samples, with shorter allele lengths being more prevalent. Lower template quality may have resulted from DNA shearing with formalin fixation [101], leading to partial repeat amplification if primer sites were unavailable for binding [102]. However, our Micro-Checker tests and other analyses demonstrate lack of statistical support for such problems. Our mtDNA sequences reveal the same pattern as the $\mu$ sat analyses. Additional analysis of historic walleye and "blue pike" samples from intermediate decades may help to further interpret temporal population genetic patterns.

\section{Taxonomic status of historic "blue pike" and turquoise-mucus variants}

We discern that the historic "blue pike" appears genetically indistinguishable from walleye populations. It has no 
unique genetic variation in our database, rendering $S$. vitreus "glaucus" invalid. It fails to meet the criteria of the Evolutionary Species Concept (ESC) [103] or the Phylogenetic Species Concept (PSC) [104]. The "blue pike" is not "an entity that kept its identity from others over time and space and that had its own independent evolutionary fate and historical tendencies" and possesses no bootstrap or posterior probability support, lacks reciprocal monophyly, diagnosable synapomorphies, and demarcation from the walleye as required by the ESC and PSC [103,104]. It also does not show interspecific variation $10 \mathrm{x}$ greater than the mean intraspecific variation of walleye [105]. In fact, the "blue pike" has no mtDNA sequence differentiation and its $\mu$ sat variation is identical to that among typical walleye spawning groups and populations. In contrast, many walleye populations across North America possess much more pronounced genetic variation, particularly from the New (X) and North Rivers (Y). Walleye spawning in those southerly locations meet more of the criteria of being distinct taxa. However, we regard those in the New and North Rivers as divergent populations of walleye, and not as separate taxa, and believe that most ichthyologists and systematists would concur. Our findings thus indicate that the "blue pike" does not constitute a separate genetic taxon from walleye, and does not merit species or subspecies recognition.

Slight morphological variations between historic walleye and "blue pike" samples suggest some possible populationlevel differences. However, single individuals of the historic "blue pike" and walleye cannot be identified morphologically or genetically as either "blue pike" or walleye. Their coloration also is not a reliable identification character as it was/is very variable among historic as well as contemporary walleye $[44,48]$. Ichthyologists from the era of the "blue pike" reported a large numbers of intergrades in color, as well as among all morphological traits $[44,48,49]$.

Some fishes, including lake trout, whitefish Coregonus spp., and Arctic char Salvelinus alpinus (Linnaeus 1758), have been regarded as multiple morphological races that developed through adaptation to northern proglacial lakes [106], but possess low genetic divergence [20]. The "blue pike" was reported to inhabit deeper waters, have slower growth [107], and a larger eye [42,this study] compared to walleye. Slower growth likewise characterizes walleye in eastern Lake Erie today [108]. We find that although "blue pike" and walleye display some slight morphological variation, this is rather negligible, and unaccompanied by population genetic distinction, rendering its subspecies status invalid. "Blue pike" were walleye, and fell within the normal range of walleye population variation.

The turquoise-colored mucus walleye from McKim Lake (site D) do not genetically differ from co-occurring yellow walleye. Stepien and Faber [41] likewise analyzed several assorted turquoise mucus walleye from a variety of Canadian Shield lakes using entire mtDNA control region sequences and found no genetic distinction from the normal variation of walleye. Paradis and Magnan [109] morphologically compared sympatric yellow and turquoise mucus walleye in five Canadian Shield lakes near Quebec, reporting longer head lengths and smaller interorbital distances in some of the latter. However, early fishery biologists found that these turquoise mucus walleye did not possess the morphological characteristics of "blue pike" [49]. Laporte et al. [110] alleged slight genetic difference between turquoise mucus and yellow walleye populations sampled within a lake using amplified fragment length polymorphism markers and assignment tests, but lacked diagnostic alleles and their genetic distance analyses showed no significant bootstrap support. It may be that there are some population-level variants within some lakes across the range of the Canadian Shield; many such distinctions among walleye spawning groups are found in the present study and others [30,39], but these do not warrant taxonomic recognition.

Occasional steel-blue colored walleye regularly are reported from Lake Erie and other waters, including the Ohio River drainage [44,48]. Yellow perch that are dark blue in color also co-occur [44]. We analyzed mtDNA control region from a steel-grey/blue walleye individual sampled in western Lake Erie near Sandusky $\mathrm{OH}$ and found it had mtDNA haplotype 1 , the most common walleye haplotype. A skin scraping revealed no turquoise mucus. Wayne Schaeffer (pers. comm., University of Wisconsin, September 2013) also found no turquoise mucus or sandercyanin in Lake Erie walleye using the methods of $\mathrm{Yu}$ et al. [47]. Overall, no diagnosable genetic or morphological characters have been found that distinguish historic "blue pike" from walleye, rendering its subspecies status invalid.

\section{Effects of climate change on walleye populations}

Global temperatures are predicted to increase over the next 50 years, with the Great Lakes region rising by $5-$ $5.5^{\circ} \mathrm{C}$, becoming more like today's Gulf Coast [111]. Today, Lake Erie houses the largest walleye abundance [45] and greatest genetic diversity. Increased temperatures are predicted to shift walleye distribution northward [23]. Fringe populations may experience declines and increased isolation, with bottlenecks and drift reducing genetic variation, accompanied by loss of unique haplotypes and alleles. Hence, valuable genetic resources may disappear as unconnected populations become sequestered.

High connectivity in Great Lakes' watersheds allows ample dispersal opportunities, which may homogenize gene pools of distinctive spawning groups as they move northward and mix, producing a Wahlund effect. Thus, 
climate change may lead to decline of divergence patterns from today's walleye spawning groups. Walleye likely will remain abundant and adapt, but unique variants may be lost. Common alleles may increase in frequency, raising concerns for retaining adaptive potential, which should become a management priority. It might be possible to utilize unique warm-adapted variants to the south (e.g., the North River, Y) and southeast (Ohio/New Rivers, $\mathrm{W}-\mathrm{X})$ to aid future walleye populations.

\section{Conclusions}

This study increases understanding of historic and contemporary walleye genetic diversity and divergence patterns via a genetic window approach. Population patterns have been shaped by climate change and drainage connections, with northern ones tracing to post-glacial recolonization. Genetic diversity and abundance of Great Lakes' walleye appear to have rebounded after drastic habitat changes and industrialization of the early 1900s through the 1970s. The next step is to identify adaptations that underlie these genetic diversity and divergence patterns, via genomics [112,113]. Such applied knowledge will aid efforts to sustain natural populations in the face of ongoing climate change and new anthropogenic stressors. Our goal is that the present results will aid future walleye diversity and adaptedness.

\section{Methods}

\section{Sample collection for genetic analysis}

Fin clips of 1125 contemporary spawning adult walleye were sampled at 23 spawning locations (labeled A-P, S-Y) across the native range during the spring spawning runs (Figure 1, Table 1, Additional file 10), avoiding sites with documented anthropogenic introductions. Samples were collected directly by federal, state, and provincial agencies according to their regulations and permits, and by Stepien's Great Lakes Genetics/Genomics Laboratory (University of Toledo's Lake Erie Center, OH) under Ohio Division of Wildlife permit \#140160 (issued to CAS). Samples were labeled, immediately placed in 95\% ethanol, and archived at the Great Lakes Genetics/ Genomics Laboratory. Genetic comparisons were made to historic formalin-preserved museum specimens of Lake Erie walleye (1923-49, site Q, $N=20$ for mtDNA, $N=31$ for $\mu$ sat) and historic "blue pike" paratype individuals (1923, $\mathrm{R}, N=20,25)$. The latter had been identified by Hubbs [42] and are housed at University of Michigan's Museum of Zoology, while some of the walleye came from Ohio State University's Museum of Biological Diversity (see Additional file 11 for list of individuals used). We also evaluated contemporary yellow$(N=10)$ versus turquoise-colored mucus $(N=10)$ walleye sympatric in McKim Lake, Ontario, Canada (site D, Figure 1).

\section{DNA extraction}

DNA was extracted following standard protocols from previous studies $[38,53]$. To circumvent contamination, museum sample extractions were conducted in a separate clean laboratory using separate autoclaved materials. Gloves were changed at all stages and between samples. Pre-extraction, formalin fixed tissues and controls were soaked in $10 \mathrm{~mL}$ phosphate buffered saline on an orbital shaker for 48 hours, changing buffer after 24 hours. Historic walleye and "blue pike" samples were extracted at separate times, with negative (no tissue) and positive controls (Lake Erie Maumee River walleye \#AZE31); the latter were handled after all solutions were added and tubes were closed.

Mitochondrial DNA sequence data collection and analyses MtDNA control region sequence data (733 bp) were collected from a representative subset from each of the 23 contemporary walleye spawning groups $(N=711$, 11-25 per site; Table 1) used for the nuclear $\mu$ sat loci. Methods for amplification and sequencing of the $733 \mathrm{bp}$ control region segment followed Haponski and Stepien [53] using the primers LW1-F [78] and HN20 [114]. We additionally sequenced formalin fixed historic Lake Erie walleye $(N=20)$ and "blue pike" $(N=20)$. Since formalin fixation often shears DNA into smaller fragments [101], we designed additional internal primers to amplify the control region in overlapping segments: LW1-F [78] and SandercrIR (5' - CATTCATACTATTTTCTTGC - 3'), SandercrIF (5' - AGTACATACTCTGTTACC - 3') with HN20 [114], SandercrIF with SvicrIR2 (5' - GTGATTTC CACTATTTATGC - 3'), and SvicrIF (5' - GCAAGAAAA TAGTATGAATG - 3') with HN20. Walleye haplotypes were referenced to Stepien and Faber [41], who sequenced the entire mtDNA control region $(\sim 1,086$ base pairs (bp)). These were trimmed to $733 \mathrm{bp}$, omitting the 5 ' repeat section, thereby retaining seven of their 14 haplotypes (our haplotypes 1-7 [GenBank:U90617, JX442946-52]).

Haplotype relationships were analyzed with evolutionary trees [50] via maximum likelihood in PhyML v3.0 [115] and Bayesian analyses in MrBayes v3.2.1 [116]. Corrected Akaike information criteria from jModeltest v2 [117] selected the TPM3uf model [118], including invariant sites $(\mathrm{I}=0.3910)$ and the gamma distribution $(\alpha=0.2750)$. Trees were rooted to the sauger $S$. canadensis (Griffith and Smith 1834), which is the sister species of walleye [52]. A statistical parsimony haplotype network was constructed with TCS V1.21 [54]. Analyses in common between the mtDNA and nuclear $\mu$ sat data sets are below in "Population genetic data analyses".

\section{Nuclear microsatellite data collection and analyses}

We evaluated population genetic structure among 23 contemporary walleye spawning groups (1125 individuals; 
Table 1, Additional file 10), adding 259 individuals and six spawning sites to the 866 individuals and 17 locations previously analyzed for nine $\mu$ sat loci (Svi2, 4, 6, 7, 17, 18, 33, L6, and L7) in our laboratory [30,38] (Additional file 11). Data were compared with museum specimens sampled from Lake Erie, including 31 walleye (1923-1949) and 25 "blue pike" (1923) (Additional file 11).

PCR amplifications followed previous studies [38,53], including a positive control and a negative control. Loci were run individually for the formalin material. Svi17 and L7 failed to amplify in historic samples and thus were dropped, leaving seven for analyses. Allele scores were manually verified $[38,53]$.

Analyses followed Stepien et al. [30] and Haponski and Stepien [53]. Conformance to HWE expectations and linkage disequilibrium was tested in Genepop v4.0 [119] and significance levels adjusted with sequential Bonferroni correction [120]. Loci were tested for possible selection using $F_{\text {ST }}$ outlier comparisons in Lositan [56] and examined for null alleles, scoring errors, or large allele dropout with Micro-Checker v2.2.3 [57]. If null alleles were indicated, their frequencies per population and locus were estimated and potential influence on $F_{\mathrm{ST}}$ values evaluated in FreeNA [58]. Number of alleles $\left(N_{\mathrm{A}}\right)$, inbreeding $\left(F_{\mathrm{IS}}\right)$, overall genetic deviation $\left(F_{\mathrm{IT}}\right)$, and divergences $\left(F_{\mathrm{ST}}\right)$ were calculated across loci and samples with $F$ stat v2.9.3.2 [121,122].

Structure v2.3.4 [61] was employed to evaluate hypotheses of differentiation among population groups, for contemporary spawning groups from $K=1$ (null hypothesis of panmixia) to $K=29$ (sites $\mathrm{A}-\mathrm{P}, \mathrm{S}-\mathrm{Y}$ ) and contemporary versus historic walleye from $K=1$ to $K=11$ (nine contemporary Lakes Erie $(\mathrm{O}-\mathrm{P})$ and Ontario $(\mathrm{S}-\mathrm{U})$ spawning groups, historic walleye (Q), and "blue pike" (R)), with 250000 burn-in replicates followed by 1000000 generations. Optimal $K$ was selected according to the Evanno et al. [123] method. Geneclass2 [59] assigned individuals to putative populations of origin for contemporary walleye, historic walleye, and "blue pike", using 1000000 simulations. Colony v2.0.5.0 [55] was employed to test for possible kin relationships (full siblings) in samples.

\section{Population genetic data analyses with both data sets}

Genetic diversity comparisons included haplotype diversity $\left(H_{\mathrm{D}}\right)$ and number of haplotypes $\left(N_{\mathrm{H}}\right)$ for sequence data in Arlequin v3.5.1.3 [124] and those for nuclear $\mu$ sat loci were observed $\left(H_{\mathrm{O}}\right)$ and expected $\left(H_{\mathrm{E}}\right)$ heterozygosities in Genepop, and $F_{\mathrm{IS}}, N_{\mathrm{A}}$, and allelic richness $\left(A_{\mathrm{R}}\right)$ in Fstat. Significant differences were determined with ANOVA in R v2.15.2 [125], followed by Tukey's post hoc tests [126]. Convert v1.31 [127] calculated number and proportion of private haplotypes $\left(N_{\mathrm{PH}}\right)$ and alleles $\left(N_{\mathrm{PA}}\right)$, i.e., those unique to a sample.

Patterns of genetic divergence were evaluated using unbiased $F_{\mathrm{ST}}$ estimates [128] in Fstat and pairwise exact tests of differentiation $\left(\chi^{2}\right)$ [129] in Genepop. Genetic divergence results were used to identify true populations or taxa, i.e., those with significantly divergent gene pools, to address the question of the identity of the "blue pike" and other possibly distinctive groups. We also tested genetic isolation by geographic distance (nearest waterways) in Genepop and identified genetic discontinuities across the range with Barrier v2.2 [26]. Population relationships additionally were analyzed with neighbor-joining trees [130] of Nei's [131] $D$ genetic distances in Phylip v3.68 [132] and 2000 bootstrap pseudo-replications [133]. The tree was rooted to sauger. Hierarchical partitioning of genetic variation was evaluated with AMOVA [60] in Arlequin, including: (a) between historic and modern samples, (b) among drainages, and (c) between lake and river spawners.

\section{Morphological comparisons}

Morphological and meristic data were collected and compared between preserved historic Lake Erie walleye $(N=51$ individuals) and "blue pike" samples $(N=52$, including 30 paratypes; all individuals are listed in Additional file 11). We compared nine morphometric measurements and five meristic characters from Hubbs and Lagler [45], using identical standard protocols taken by a single investigator (AEH). Measurements (to $0.1 \mathrm{~mm}$ ) included: standard, head, cheek, upper and lower jaw lengths, body depth, head and interorbital widths, and orbit diameter, with Mitutoyo vernier calipers (Aurora, IL). Measurements were taken to the bone of individual fish, to rule out influence of preservation technique. Meristic characters included: numbers of anal, first and second dorsal, pectoral, and pelvic fin rays, with a Leica Microsystems dissecting microscope (Buffalo Grove, IL). We also analyzed orbit: interorbital and upper: lower jaw ratios, reported by Trautman [44] to vary between walleye and "blue pike".

Morphometric measurements first were standardized by removing size-dependent variation per Elliott et al. [134], and then log-transformed. Meristic data were not transformed; therefore morphometric and meristic data sets were analyzed separately, encompassing univariate and multivariate analyses in R. Each character was evaluated for departure from univariate normality with ShapiroFrancia tests [135]. Standard length, body depth, and orbit: interorbital ratio were the sole normally distributed characters. Their means were compared with Student's $t$-tests, whereas Mann-Whitney $U$ tests evaluated those that were not normally-distributed [126]. Nonparametric MANOVA [136] compared the overall difference between historic walleye and "blue pike". Their overall morphological variation also was explored with PCA, using the covariance matrix. MANOVA and Wilks' lambda [126] tests evaluated among PCs, followed by individual ANOVAs. 
DFA [137] compared historic walleye and "blue pike" in SAS v9.2 (SAS Institute Inc., Cary, NC). $\chi^{2}$ evaluated use of linear or quadratic DFA for significant covariance matrix heterogeneity, rejecting linear DFA (morphometric: $\chi^{2}=103.216, \mathrm{df}=45, p<0.001 ;$ meristic: $X^{2}=32.253$, $\mathrm{df}=15, p=0.006)$. Thus, quadratic DFA was used, which based each character on its own variance-covariance matrix, with leave-one-out jackknife re-sampling examining taxon assignment [138].

\section{Data set availability}

The data sets supporting the results of this article are available in the Dryad digital repository [http://doi.org/10.5061/ dryad.nk470] [139] and GenBank:U90617, JX442946-56, KC819843-54, and KF954732-35.

\section{Additional files}

Additional file 1: Phylogenetic tree of contemporary and historic walleye mtDNA control region haplotypes. Above branches = Bayesian posterior probabilities (pp) and Maximum likelihood bootstrap pseudoreplications. Nodes with $\geq 0.50 \mathrm{pp}$ and $\geq 50 \%$ bootstrap support are reported. * haplotypes found in historic walleye and "blue pike" samples; the latter had no unique haplotypes. All "blue pike" individuals had haplotype 3, which is one of the most common walleye haplotypes.

Additional file 2: MtDNA control region haplotype frequencies for population samples, including 23 contemporary walleye spawning groups, historic Lake Erie walleye, and "blue pike".

Additional file 3: Summarized genetic variation per microsatellite locus for population samples, including 23 contemporary walleye spawning groups, historic walleye, and "blue pike" samples, totaling 1181 individuals. Table shows PCR annealing temperature $\left(T_{A}\right)$ number of alleles $\left(N_{A}\right)$, allelic size range (base pairs, bp), genetic deviation across all combined samples $\left(F_{I_{T}}\right)$, mean genetic divergence among loci $\left(F_{\mathrm{ST}}\right)$, inbreeding coefficient $\left(F_{\mathrm{IS}}\right.$, average divergence within a spawning group), and neutrality test from the program Lositan [56].

Additional file 4: Allelic sizes and distribution for seven nuclear $\mu$ sat loci among samples, including: contemporary walleye from Lakes Erie (the western and eastern basins) and Ontario, historic Lake Erie walleye, and the "blue pike". Site labels (letters) match those in Table 1.

Additional file 5: Genetic isolation by geographic distance comparison among $\mathbf{2 3}$ contemporary spawning groups of walleye. A mtDNA control region $\left(y=0.64 x-3.67, R^{2}=0.10, p<0.001\right)$ and $\mathbf{B}$ seven nuclear $\mu$ sat loci $\left(y=0.06 x-0.29, R^{2}=0.23, p=0.005\right)$. Results from the seven loci are identical to those for nine loci (data not shown; see Stepien et al. [30]). Letters correspond to spawning group labels from Table 1.

Additional file 6: Pairwise exact tests of genetic differentiation among population samples (lettered) for control region sequence data (below diagonal) and seven nuclear $\mu$ sat loci (above diagonal). Results are congruent to $F_{\text {ST }}$ comparisons and those from the seven nuclear usat loci data are identical to values calculated based on nine loci (data not shown; also see Stepien et al. [30]), with difference at just the thousandth decimal place. Inf. = Infinite value denoted by Genepop [119], bold = significant following sequential Bonferroni corrections, italics = significant at $a=0.05$, and normal text $=$ not significant.

Additional file 7: Geneclass2 [59] assignments among contemporary walleye spawning groups. Values = percentage assignment, parentheses $=$ number of individuals assigning to that group, bold $=$ self-assignment, and italics $=$ greatest assignment. Numbers in parentheses next to spawning group denote sample size.
Additional file 8: Graph of $K$ vs. $\Delta K$ based on Evanno et al. [123], showing the optimal $K$ A contemporary walleye spawning groups and B contemporary Lakes Erie and Ontario and historic Lake Erie walleye (with "blue pike").

Additional file 9: Population genetic distance neighbor joining tree for contemporary walleye Sander vitreus vitreus spawning groups, historic walleye, and "blue pike" S. v. "glaucus" in relation to sauger S. canadensis. Tree is based on combined frequencies of mtDNA control region haplotypes and alleles from the seven nuclear $\mu$ sat loci.

Additional file 10: Latitude (Lat.) and longitude (Long.) for each contemporary walleye spawning group used in analyses. Letters correspond to locations from Figure 1 and Table 1.

Additional file 11: Materials examined, including A museum specimens and B additional contemporary specimens that augmented prior work reported by Stepien et al. (2009, 2010, 2012). Institutional abbreviations follow Leviton et al. [140]. Sample information is listed as follows: museum - lot number, number of samples, size range $(\mathrm{SL}, \mathrm{mm})$, locality, sampling date, and collector. Individuals that also were analyzed with molecular data are listed in the "Molecular Samples" section with their respective lot numbers and GenBank accession numbers. Individuals analyzed solely for molecular data are listed under "Molecular Samples" with lot number, number of samples, locality, sampling date, collector, and GenBank accession number.

\section{Competing interests}

The authors declare that they have no competing interests.

\section{Authors' contributions}

CAS conceived the project. AEH collected, analyzed, and interpreted the molecular and morphological data, drafted, and revised the paper. CAS oversaw the project design, assisted in data interpretation, added major parts and revisions to the paper, and extensively edited it. Both authors have read and approved the final manuscript.

\section{Authors' information}

This work formed part of the Ph.D. dissertation work of AEH in the laboratory of CAS, who now is a postdoctoral associate in the laboratory. CAS has worked on walleye genetic patterns for over two decades, and her Great Lakes Genetics/Genomics laboratory focuses on invasive species, as well as native fishes.

\section{Acknowledgements}

This is contribution \#2014-009 from the University of Toledo's Lake Erie Research Center. Research was funded by grants to CAS from NOAA Ohio Sea Grant R/LR-013 and USEPA CR-83281401-0. Travel by AEH was supplemented by NSF Deepfin \#RCN-0443470 (P.I. = G. Orti), a Smithsonian Fellowship visiting appointment (sponsored by L. Parenti), and a Sigma Xi Grant-In-Aid of Research. AEH also was supported by NSF GK-12 DGE\#0742395 fellowship (P.I. = CAS), University of Toledo teaching and research assistantships, and Norman S. Baldwin Fishery Science (2010) and IAGLR (International Association for Great Lakes Research) Scholarships (2009). The U.S. Fish and Wildlife Service, U.S. Geological Survey, Ohio and Michigan Departments of Natural Resources, Ontario Ministry of Natural Resources, and Keweenaw Bay Indian Community Natural Resources Department, M. Bagley, L. Bernatchez, N. Billington, E. Hallerman, W. Jansen, M. Kibbey, G. Mensch, D. Nelson, W. Schaefer, C. Wentzel, M. White, and C. Wilson contributed samples. We thank J. Bossenbroek, T. Fisher, J. Gottgens. P. Kocovsky, L. Parenti, W. Stott, and Great Lakes Genetics/Genomics Laboratory members L. Pierce, C. Prichard, T. Sullivan, and S. Yerga-Woolwine for assistance, and P. Uzmann, M. Gray, P. Struffolino, and R. Lohner for logistic support.

Received: 28 October 2013 Accepted: 5 June 2014 Published: 17 June 2014 


\section{References}

1. Dawson TP, Jackson ST, House Jl, Prentice IC, Mace GM: Beyond predictions: biodiversity conservation in a changing climate. Science 2011, 332:53-58.

2. Hutchings JA, Butchart SHM, Collen B, Schwartz MK, Waples RS: Red flags: correlates of impaired species recovery. Trends Ecol Evol 2012, 27:542-546.

3. Reed DH, Frankham R: Correlation between fitness and genetic diversity. Conserv Biol 2003, 17:230-237.

4. Väli U, Einarsson A, Waits $L$, Ellegren $H$ : To what extent do microsatellite markers reflect genome-wide genetic diversity in natural populations? Mol Ecol 2008, 17:3808-3817.

5. Taubmann J, Theissinger K, Feldheim KA, Laube I, Graf W, Haase P, Johannesen J, Pauls SU: Modeling range shifts and assessing genetic diversity distribution of the montane aquatic mayfly Ameletus inopinatus in Europe under climate change scenarios. Conserv Genet 2011, 12:503-515.

6. Allendorf FW, Luikart G, Aitken SN: Conservation and the genetics of populations. 2nd edition. Oxford, UK: Blackwell Publishing; 2013.

7. Davis MB, Shaw RG: Range shifts and adaptive responses to quaternary climate change. Science 2001, 292:673-679.

8. Taylor EB, Gow JL, Witt J, Zemlak R: Connectivity among populations of pygmy whitefish (Prosopium coulterii) in northwestern North America inferred from microsatellite DNA analyses. Can J Zool 2011, 89:255-266.

9. Carvalho GR: Evolutionary aspects of fish distribution: genetic variability and adaptation. J Fish Biol 1993, 43:53-73.

10. Ozerov MY, Veselov AE, Lumme J, Primmer CR: "Riverscape" genetics: river characteristics influence the genetic structure and diversity of anadromous and freshwater Atlantic salmon (Salmo salar) populations in northwest Russia. Can J Fish Aquat Sci 2012, 69:1947-1958.

11. Slatkin M: Gene flow in natural populations. Annu Rev Ecol Syst 1985, 16:393-430

12. Wofford JEB, Gresswell RE, Banks MA: Influence of barriers to movement on within-watershed genetic variation of coastal cutthroat trout. Ecol Appl 2005, 15:628-637.

13. Moran GF, Hopper SD: Genetic diversity and the insular population structure of the rare granite rock species, Eucalyptus caesia Benth. Aust J Bot 1983, 31:161-172.

14. Coulon A, Fitzpatrick JW, Bowman R, Lovette IJ: Mind the gap: genetic distance increases with habitat gap size in Florida scrub jays. Biol Lett 2012, 8:582-585.

15. Poissant J, Knight TW, Ferguson MM: Nonequilibrium conditions following landscape rearrangement: the relative contribution of past and current hydrological landscapes on the genetic structure of a stream-dwelling fish. Mol Ecol 2005, 14:1321-1331.

16. Huey JA, Baker AM, Hughes JM: High levels of genetic structure in the Australian freshwater fish, Ambassis macleayi. J N Am Benthol Soc 2010, 29:1148-1160.

17. Waples RS: A multispecies approach to the analysis of gene flow in marine shore fishes. Evolution 1987, 41:385-400.

18. Pringle JM, Wares JP: Going against the flow: maintenance of alongshore variation in allele frequency in a coastal ocean. Mar Ecol-Prog Ser 2007, 335:69-84.

19. Hewitt GM: Some genetic consequences of ice ages, and their role in divergence and speciation. Biol J Linn Soc 1996, 58:247-276.

20. Bernatchez L, Wilson CC: Comparative phylogeography of Nearctic and Palearctic fishes. Mol Ecol 1998, 7:431-452.

21. Ward RD, Billington N, Hebert PDN: Comparison of allozyme and mitochondrial DNA variation in populations of walleye, Stizostedion vitreum. Can J Fish Aquat Sci 1989, 46:2074-2084.

22. Billington N, Barrette RJ, Hebert PDN: Management implications of mitochondrial DNA variation in walleye stocks. N Am J Fish Manage 1992, 12:276-284

23. Chu C, Mandrak NE, Minns CK: Potential impacts of climate change on the distributions of several common and rare freshwater fishes in Canada. Divers Distrib 2005, 11:299-310.

24. Sharma S, Jackson DA, Minns CK, Shuter BJ: Will northern fish populations be in hot water because of climate change? Glob Change Biol 2007, 13:2052-2064.

25. Billington N, Wilson C, Sloss BL: Distribution and population genetics of walleye and sauger. In Biology, management, and culture of Walleye and Sauger. Edited by Barton BA. Bethesda, MD: American Fisheries Society; 2011:105-132.
26. Manni F, Guérard E, Heyer E: Geographic patterns of (genetic, morphologic, linguistic) variation: how barriers can be detected by using Monmonier's algorithm. Hum Biol 2004, 76:173-190. Available at http:// www.mnhn.fr/mnhn/ecoanthropologie/software/barrier.html.

27. Faulks LK, Gilligan DM, Beheregaray LB: The role of anthropogenic vs. natural in-stream structures in determining connectivity and genetic diversity in an endangered freshwater fish, Macquarie perch (Macquaria australasica). Evol Appl 2011, 4:589-601.

28. Williams JE, Haak AL, Neville HM, Colyer WT: Potential consequences of climate change to persistence of cutthroat trout populations. N Am J Fish Manage 2009, 29:533-548.

29. Jonsson $B$, Jonsson $\mathrm{N}$ : A review of the likely effects of climate change on anadromous Atlantic salmon Salmo salar and brown trout Salmo trutta, with particular reference to water temperature and flow. J Fish Biol 2009, 75:2381-2447

30. Stepien CA, Murphy DJ, Lohner RN, Sepulveda-Villet OJ, Haponski AE: Signatures of vicariance, postglacial dispersal, and spawning philopatry: population genetics and biogeography of the walleye Sander vitreus. Mol Ecol 2009, 18:3411-3428.

31. Hauser L, Adcock GJ, Smith PJ, Ramírez JHB, Carvalho GR: Loss of microsatellite diversity and low effective population size in an overexploited population of New Zealand snapper (Pagrus auratus). PNAS 2002, 99:11742-11747.

32. Locke B, Belore M, Cook A, Einhouse D, Kenyon R, Knight R, Newman K, Ryan P, Wright E Walleye management plan: Lake Erie Committee Great Lakes Fishery Commission. 2005. Available at http://www.glfc.org/lakecom/ lec/WTG_docs/other_reports_and_docs/wmp20051207.pdf.

33. Nate NA, Hansen MJ, Rudstam LG, Knight RL, Newman SP: Population and community dynamics of walleye. In Biology, management, and culture of Walleye and Sauger. Edited by Barton BA. Bethesda, MD: American Fisheries Society; 2011:321-374.

34. Schmalz PJ, Fayram AH, Isermann DA, Newman SP, Edwards CJ: Harvest and exploitation. In Biology, management, and culture of Walleye and Sauger. Edited by Barton BA. Bethesda, MD: American Fisheries Society; 2011:375-401.

35. Barton BA, Barry TP: Reproduction and environmental biology. In Biology, management, and culture of Walleye and Sauger. Edited by Barton BA. Bethesda, MD: American Fisheries Society; 2011:199-231.

36. Jennings MJ, Claussen JE, Philipp DP: Evidence for heritable preferences for spawning habitat between two walleye populations. TAm Fish Soc 1996, 125:978-986.

37. Bozek MA, Baccante DA, Lester NP: Walleye and sauger life history. In Biology, management, and culture of Walleye and Sauger. Edited by Barton BA. Bethesda, MD: American Fisheries Society; 2011:233-301.

38. Stepien CA, Banda JA, Murphy DJ, Haponski AE: Temporal and spatial genetic consistency of walleye (Sander vitreus) spawning groups. TAm Fish Soc 2012, 141:660-672.

39. Strange RM, Stepien CA: Genetic divergence and connectivity among river and reef spawning groups of walleye (Sander vitreus) in Lake Erie. Can J Fish Aquat Sci 2007, 64:437-448.

40. Stepien CA, Murphy DJ, Lohner RN, Haponski AE, Sepulveda-Villet OJ: Status and delineation of walleye (Sander vitreus) genetic stock structure across the Great Lakes. In Status of walleye in the Great Lakes: proceedings of the 2006 symposium. Edited by Roseman E, Kocovsky P, Vandergoot C. Ann Arbor, Ml: Great Lakes Fishery Commission Technical Report 69; 2010:189-223.

41. Stepien CA, Faber JE: Population genetic structure, phylogeography, and spawning philopatry in walleye (Stizostedion vitreum) from mtDNA control region sequences. Mol Ecol 1998, 7:1757-1769.

42. Hubbs CL: A check-list of the fishes of the Great Lakes and tributary waters, with nomenclatorial notes and analytical keys. Ann Arbor, Ml: University of Michigan Museum of Zoology Miscellaneous Publication; 1926:15.

43. Stone FL: A study of the taxonomy of the blue and yellow pikeperches (Stizostedion) of Lake Erie and Lake Ontario, Unpublished Ph.D. dissertation. Rochester, NY: University of Rochester; 1948.

44. Trautman MB: The fishes of Ohio. Columbus, OH: Ohio State University Press; 1981.

45. Hubbs CL, Lagler KF: Fishes of the Great Lakes Region. (G.R. Smith, revised). Ann Arbor, Ml: University of Michigan; 2004.

46. Noecker RJ: Endangered species list revisions: a summary of delisting and downlisting, CRS Report for Congress 98-32 ENR. ; 1998. 
47. Yu C, Ferraro D, Ramaswamy S, Schmitz MH, Schaefer WF, Gibson DT: Purification and properties of sandercyanin, a blue protein secreted in the mucus of blue forms of walleye, Sander vitreus. Environ Biol Fish 2008, 82:51-58.

48. Scott WB, Crossman EJ: Freshwater fishes of Canada. Fish Res Board Can 1973, 184:1-196

49. Campbell RR: Status of the blue walleye, Stizostedion vitreum glaucum, in Canada. Can Field Nat 1987, 101:245-252.

50. Avise JC: Molecular markers, natural history, and evolution. 2nd edition. Sunderland, MA: Sinauer Associates; 2004.

51. Wang IJ: Recognizing the temporal distinctions between landscape genetics and phylogeography. Mol Ecol 2010, 19:2605-2608.

52. Haponski AE, Stepien CA: Phylogenetic and biogeographic relationships of the Sander pikeperches (Perciformes: Percidae): patterns across North America and Eurasia. Biol J Linn Soc 2013, 110:156-179.

53. Haponski AE, Stepien CA: Genetic connectivity and diversity of walleye (Sander vitreus) spawning groups in the Huron-Erie Corridor. J Great Lakes Res 2014, 49(2014):89-100.

54. Clement M, Posada D, Crandall KA: TCS: a computer program to estimate gene genealogies. Mol Ecol 2000, 9:1657-1660. Available at http://darwin. uvigo.es/software/tcs.html.

55. Jones O, Wang J: COLONY: a program for parentage and sibship inference from multilocus genotype data. Mol Ecol Resour 2009, 10:551-555. Available at http://www.zsl.org/science/software/colony

56. Antao T, Lopes A, Lopes RJ, Beja-Pereira A, Luikart G: LOSITAN: a workbench to detect molecular adaptation based on a Fst-outlie method. BMC Bioinformatics 2008, 9:323. Available at http://popgen.net/ soft/lositan/.

57. van Oosterhout C, Hutchinson WF, Wills DPM, Shipley P: Micro-checker: software for identifying and correcting genotyping errors in microsatellite data. Mol Ecol Notes 2004, 4:535-538. Available at http://www.microchecker.hull.ac.uk/

58. Chapuis M, Estoup A: Microsatellite null alleles and estimation of population differentiation. Mol Biol Evol 2007, 24:621-631.

59. Piry S, Alapetite A, Cornuet JM, Paetkau D, Baudouin L, Estoup A: Geneclass2: a software for genetic assignment and first-generation migrant detection. J Hered 2004, 95:536-539. Available at http://www. ensam.inra.fr/URLB/index.html.

60. Excoffier L, Smouse P, Quattro J: Analysis of molecular variance inferred from metric distances among DNA haplotypes: application to human mitochondrial DNA restriction data. Genetics 1992, 131:479-491.

61. Pritchard JK, Stephens M, Donnelly P: Inference of population structure using multilocus genotype data. Genetics 2000, 155:945-959. Available at http://pritchardlab.stanford.edu/structure_software/release_versions/v2.3.4/ html/WhatsNew.html.

62. Palmer GC, Culver C, Dutton D, Murphy BR, Hallerman EM, Billington N, Williams J: Genetic distinct walleye stocks in Claytor Lake and the Upper New River, Virginia. P Southeast Fish Wild Agencies 2006, 60:125-131.

63. Danzmann RG, Morgan RP, Jones MW, Bernatchez L, Ihssen PH: A major sextet of mitochondrial DNA phylogenetic assemblages extant in eastern North American brook trout (Salvelinus fontinalis): distribution and postglacial dispersal patterns. Can J Fish Aquat Sci 1998, 76:1300-1318.

64. Stepien CA, Murphy DJ, Strange RM: Broad- to fine-scale population genetic patterning in the smallmouth bass Micropterus dolomieu across the Laurentian Great Lakes and beyond: an interplay of behaviour and geography. Mol Ecol 2007, 16:1605-1624.

65. Haponski AE, Stepien CA: Molecular, morphological, and biogeographic resolution of cryptic taxa in the greenside darter Etheostoma blennioides complex. Mol Phylogenet Evol 2008, 49:69-83.

66. Haponski AE, Stepien CA: Landscape genetic patterns of the rainbow darter: a watershed analysis of mitochondrial DNA sequences and nuclear microsatellites. J Fish Biol 2009, 75:2244-2268.

67. Sepulveda-Villet OJ, Stepien CA: Waterscape genetics of the yellow perch (Perca flavescens): patterns across large connected ecosystems and isolated relict populations. Mol Ecol 2012, 21:5795-5826.

68. Boschung HT, Mayden RL: Fishes of Alabama. Washington D.C: Smithsonian Institution; 2004.

69. Walter RP, Cena CJ, Morgan GE, Heath DD: Historical and anthropogenic factors affecting the population genetic structure of Ontario's inland lake populations of walleye (Sander vitreus). J Hered 2012, 103:831-841.
70. Karsiotis SI, Sullivan TJ, Stepien CA: Population genetic structure and comparative diversity of smallmouth bass: patterns from two genomes. J Fish Biol. In review.

71. Rawson MR: Yellow perch movements. Columbus, OH: Ohio Department of Natural Resources Job Program Report. Dingell-Johnson Project Number F-35-R-18, Study Number. 4, July 1, 1979-June 30, 1980.

72. Lyons J, Kanehl P: Seasonal movements of smallmouth bass in streams. In Black bass: ecology, conservation, and management. Edited by Philipp DP Ridgway MS. Bethesda, MD: American Fisheries Society; 2002:149-160.

73. Colby PJ, McNicol RE, Ryder RA: Synopsis of biological data on the walleye Stizostedion v. vitreum. Rome, IT: FAO Fisheries Synopsis; 1979:119.

74. Gerlach G, Schardt U, Eckmann R, Meyer A: Kin-structured subpopulations in Eurasian perch (Perca fluviatilis L.). Heredity 2001, 86:213-221.

75. Behrmann-Godel J, Gerlach G: First evidence for postzygotic reproductive isolation between two populations of Eurasian perch (Perca fluviatilis L.) within Lake Constance. Front Zool 2008, 5:1-7.

76. Sullivan TJ, Stepien CA: Temporal population genetic structure of the yellow perch Perca flavescens (Percidae: Teleostei) within a complex lakescape. TAm Fish Soc. In review.

77. Hewitt GM: Speciation, hybrid zones and phylogeography - or seeing genes in space and time. Mol Ecol 2001, 10:537-549.

78. Gatt MH, Fraser DJ, Liskauskas AP, Ferguson MM: Mitochondrial DNA variation and stock structure of walleye from eastern Lake Huron: an analysis of contemporary and historical samples. T Am Fish Soc 2002, 131:99-108.

79. Nesbø CL, Fossheim T, Vollestad LA, Jakobsen KS: Genetic divergence and phylogeographic relationships among European perch (Perca fluviatilis) populations reflect glacial refugia and postglacial colonization. Mol Ecol 1999, 8:1387-1404.

80. Stepien CA, Dillon AK, Chandler MD: Genetic identity, phylogeography, and systematics of ruffe Gymnocephalus in the North American Great Lakes and Eurasia. J Great Lakes Res 1998, 24:361-378.

81. Hartman WL: The effects of exploitation, environmental changes, and new species on the fish habitats and resources of Lake Erie. Ann Arbor, Ml: Great Lakes Fishery Commission Technical Report 22; 1973.

82. Hartig JH, Zarull MA, Ciborowski JJH, Gannon JE, Wilke E, Norwood G, Vincent AN: Long-term ecosystem monitoring and assessment of the Detroit River and western Lake Erie. Environ Monit Assess 2009, 158:87-104.

83. Bennion DH, Manny BA: Construction of shipping channels in the Detroit River-history and environmental consequences. Reston, VA: U.S. Geological Survey Scientific Investigations Report 2011-5122; 2011

84. Ryan PA, Knight R, MacGregor R, Towns G, Hoopes R, Culligan W: Fish-community goals and objectives for Lake Erie. Ann Arbor, Ml: Great Lakes Fishery Commission Special Publication 03-02; 2003.

85. Manny BA, Kenaga D: The Detroit River: effects of contaminants and human activities on aquatic plants and animals and their habitats. Hydrobiologia 1991, 219:269-279.

86. Guinand B, Scribner KT, Page KS, Burnham-Curtis MK: Genetic variation over space and time: analyses of extinct and remnant lake trout populations in the Upper Great Lakes. P R Soc London B 2003, 270:425-433.

87. Stott W, Ebener MP, Mohr L, Hartman T, Johnson J, Roseman EF: Spatial and temporal genetic diversity of lake whitefish (Coregonus clupeaformis (Mitchill)) from Lake Huron and Lake Erie. Adv Limnol 2013, 64:205-222.

88. Hutchinson WF, van Oosterhout C, Rogers SI, Carvalho GR: Temporal analysis of archived samples indicates marked genetic changes in declining North Sea Cod (Gadus morhua). P R Soc London B 2003, 270:2125-2132.

89. Olson DE, Scidmore WJ: Homing behavior of spawning walleyes. TAm Fish Soc 1962, 91:355-361.

90. Colby PJ, Nepszy SJ: Variation among stocks of walleye (Stizostedion vitreum vitreum): management implications. Can J Fish Aquat Sci 1981, 38:1814-1831

91. Haas RC, Bryant WC, Smith KD, Nuhfer AJ: Movement and harvest of fish in Lake St. Clair, St. Clair River, and Detroit River. Final Report Winter Navigation Study U.S. Army Corps of Engineers: Detroit, Ml; 1985

92. Wang HY, Rutherford ES, Cook HA, Einhouse DW, Haas RC, Johnson TB, Kenyon R, Locke B, Turner MW: Movement of walleye in Lakes Erie and St. Clair inferred from tag return and fisheries data. T Am Fish Soc 2007, 136:539-551.

93. Haas RC, Thomas MV: The walleye population of Lake Erie. In State of the strait: status and trends of key indicators. Edited by Hartig JH, Zarull MA, 
Ciborowski JJH, Gannon JE, Wilke E, Norwood G, Vincent A. Windsor, ON Great Lakes Institute for Environmental Research Occasional Publication No. 5; 2007:226-229

94. WTG (Walleye Task Group of the Lake Erie Committee, Great Lakes Fishery Commission): Report for 2012 by the Lake Erie Walleye task group. Ann Arbor, Ml: Great Lakes Fishery Commission; 2013. Available at http://www.glfc.org/ lakecom/lec/WTG_docs/annual_reports/WTG_report_2013.pdf.

95. Franckowiak RP, Sloss BL, Bozek MA, Newman SP: Temporal effective size estimates of a managed walleye Sander vitreus population and implications for genetic-based management. J Fish Biol 2009, 74:1086-1103.

96. Garner SW, Bobrowicz SM, Wilson CC: Genetic and ecological assessment of population rehabilitation: Walleye in Lake Superior. Ecol App/ 2013, 23:594-605.

97. Waples RS: Conservation genetics of Pacific salmon. III. Estimating effective population size. J Hered 1990, 81:277-289.

98. Nielsen $\mathrm{EE}$, Hansen MM: Waking the dead: the value of population genetic analyses of historical samples. Fish Fish 2008, 9:450-461.

99. Taberlet P, Griffin S, Goossens B, Questiau S, Manceau V, Escaravage N, Waits LP, Bouvet J: Reliable genotyping of samples with very low DNA quantities using PCR. Nucleic Acids Res 1996, 24:3189-3194.

100. DeWoody J, Nason JD, Hipkins VD: Mitigating scoring errors in microsatellite data from wild populations. Mol Ecol Notes 2006, 6:951-957.

101. Shedlock AM, Haygood MG, Pietsch TW, Bentz P: Enhanced DNA extraction and PCR amplification of mitochondrial genes from formalin fixed museum specimens. Biotechniques 1997, 22:394-400.

102. Ugelvig LV, Nielsen PS, Boomsma JJ, Nash DR: Reconstructing eight decades of genetic variation in an isolated Danish population of the large blue butterfly Maculinea arion. BMC Evol Biol 2011, 11:201.

103. Wiley EO, Mayden RL: The Evolutionary Species Concept. In Species concepts and phylogenetic theory, a debate. Edited by Wheeler QD, Meier R. New York, NY: Columbia University Press; 2000:70-89.

104. Mishler BD, Theriot EC: The phylogenetic species concept (sensu Mishler and Theriot): Monophyly, apomorphy, and phylogenetic species concepts. In Species concepts and phylogenetic theory, a debate. Edited by Wheeler QD, Meier R. New York, NY: Columbia University Press; 2000:44-54.

105. Hebert PDN, Penton EH, Burns JM, Danzen DH, Hallwachs W: Ten species in one: DNA barcoding reveals cryptic species in the neotropical skipper butterfly Astraptes fulgerator. PNAS 2004, 101:14812-14817.

106. Robinson BW, Parsons KJ: Changing times, spaces, and faces: tests and implications of adaptive morphological plasticity in the fishes of northern postglacial lakes. Can J Fish Aquat Sci 2002, 59:1819-1833.

107. Parsons JW: Contributions of year-classes of blue pike to the commercial fishery of Lake Erie, 1943-59. Fish Res Board Can 1967, 24:1035-1066.

108. Einhouse DW, MacDougall TM: An emerging view of the mixed-stock structure of Lake Erie's eastern-basin walleye population. In Status of walleye in the Great Lakes: proceedings of the 2006 symposium. Edited by Roseman E, Kocovsky P, Vandergoot C. Ann Arbor, Ml: Great Lakes Fishery Commission Technical Report 69; 2010:151-164.

109. Paradis Y, Magnan P: Phenotypic variation of walleye, Sander vitreus, in Canadian Shield lakes: new insights on percid polymorphism. Env Biol Fish 2005, 73:357-366.

110. Laporte M, Magnan P, Angers B: Genetic differentiation between the blue and the yellow phenotypes of walleye (Sander vitreus): an example of parallel evolution. Ecoscience 2011, 18:124-129.

111. Hayhoe K, VanDorn J, Croley T, Schlegal N, Wuebbles D: Regional climate change projections for Chicago and the US Great Lakes. J Great Lakes Res 2010, 36:7-21.

112. Allendorf FW, Hohenlohe PA, Luikart G: Genomics and the future of conservation genetics. Nat Rev Genet 2010, 11:697-710.

113. Bradbury IR, Hubert S, Higgins B, Bowman S, Borza T, Paterson IG, Snelgrove PVR, Morris CJ, Gregory RS, Hardie D, Hutchings JA, Ruzzante DE, Taggart CT, Bentzen P: Genomic islands of divergence and their consequences for the resolution of spatial structure in an exploited marine fish. Evol Appl 2013, 6:450-461

114. Bernatchez L, Danzmann RG: Congruence in control-region sequence and restriction-site variation in mitochondrial DNA of brook charr (Salvelinus fontinalis Mitchill). Mol Biol Evol 1993, 10:1002-1014.

115. Guindon S, Dufayard JF, Lefort V, Anisimova M, Hordijk W, Gascuel O: New Algorithms and methods to estimate maximum-likelihood phylogenies: assessing the performance of PhyML 3.0. Systematic Biol 2010, 59:307-321. Available at http://www.atgc-montpellier.fr/phyml/.
116. Ronquist F, Huelsenbeck JP: MrBayes 3: Bayesian phylogenetic inference under mixed models. Bioinformatics 2003, 19:1572-1574. http://mrbayes. csit.fsu.edu/(v3.1.2, 2005).

117. Darriba D, Taboada GL, Doallom R, Posada D: jModelTest 2: more models, new heuristics and parallel computing. Nat Methods 2012, 9:772. Available at http://code.google.com/p/jmodeltest2/.

118. Posada D: jModelTest: phylogenetic model averaging. Mol Biol Evol 2008, 25:1253-1256. Available at http://code.google.com/p/jmodeltest2/.

119. Rousset F: Genepop'008: a complete re-implementation of the Genepop software for Windows and Linux. Mol Ecol Resour 2008, 8:103-106. Available at http://kimura.univ-montp2.fr/ rousset/Genepop.htm.

120. Rice RM: Analyzing tables of statistical tests. Evolution 1989, 43:223-225.

121. Goudet J: Fstat version 1.2: a computer program to calculate Fstatistics. $J$ Hered 1995, 86:485-486.

122. Goudet J: Fstat version 2.9.3.2. 2002. Available at http://www2.unil.ch/ popgen/softwares/fstat.htm.

123. Evanno G, Regnaut S, Goudet J: Detecting the number of clusters of individuals using the software structure: a simulation study. Mol Ecol 2005, 14:2611-2620

124. Excoffier L, Lischer HE: Arlequin suite ver 3.5: a new series of programs to perform population genetics analyses under Linux and Windows. Mol Ecol Resour 2010, 10:564-567. Available at http://cmpg.unibe.ch/software/ arlequin35/.

125. R Development Core Team: $R$ : a language and environment for statistical computing. Vienna, IT: R Foundation for Statistical Computing; 2012. Available at http://www.R-project.org.

126. Zar JH: Biostatistical analysis. 4th edition. Upper Saddle River, NJ: Prentice Hall; 1999.

127. Glaubitz JC: CONVERT: a user-friendly program to reformat diploid genotypic data for commonly used population genetic software packages. Mol Ecol Notes 2004, 4:309-310. http://www.agriculture.purdue. edu/fnr/html/faculty/rhodes/students\%20and\%20staff/glaubitz/software.htm.

128. Weir BS, Cockerham CC: Estimating F-statistics for the analysis of population structure. Evolution 1984, 38:1358-1370.

129. Raymond M, Rousset F: An exact test for population differentiation. Evolution 1995, 49:1280-1283

130. Saitou N, Nei M: The neighbor joining method: a new method for reconstructing phylogenetic trees. Mol Biol Evol 1987, 4:406-425.

131. Nei M: Genetic distance between populations. Am Nat 1972, 106:283-282.

132. Felsenstein J: Phylip (Phylogeny Inference Package) version 3.6. Distributed by the author. Seattle: Department of Genome Sciences, University of Washington; 2005. http://evolution.genetics.washington.edu/phylip.html.

133. Felsenstein J: Confidence limits on phylogenies: an approach using the bootstrap. Evolution 1985, 39:783-791.

134. Elliott NG, Haskard K, Koslow JA: Morphometric analysis of orange roughy (Hoplostethus atlanticus) off the continental slope of southern Australia. J Fish Biol 1995, 46:202-220.

135. Royston P: A pocket-calculator algorithm for the Shapiro-Francia test for non-normality: an application to medicine. Stat Med 1993, 12:181-184.

136. Anderson MJ: A new method for non-parametric multivariate analysis of variance. Austral Ecol 2001, 26:32-46.

137. dos Reis SF, Pessôa LM, Strauss RE: Application of size-free canonical discriminant analysis to studies of geographic differentiation. Braz J Genet 1990, 13:509-520.

138. McGarigal K, Cushman S, Stafford S: Multivariate statistics for wildlife and ecology research. New York, NY: Springer-Verlag; 2000.

139. Haponski AE, Stepien CA: Nuclear DNA microsatellite loci and mitochondrial DNA sequence data from walleye Sander vitreus. Dryad; 2014 http://doi.org/10.5061/dryad.nk470.

140. Leviton AE: Standards in herpetology and ichthyology, part 1. Standard symbolic codes for institutional resource collections in herpetology and ichthyology. Copeia 1985, 1985:802-832.

doi:10.1186/1471-2148-14-133

Cite this article as: Haponski and Stepien: A population genetic window into the past and future of the walleye Sander vitreus: relation to historic walleye and the extinct "blue pike" S. v. "glaucus". BMC Evolutionary Biology 2014 14:133. 